\title{
Star Polymers and the Failure of Time-Temperature Superposition
}

\author{
Alex J . Levine* and Scott T. Milner \\ Exxon Research and Engineering, Route 22 East, Annandale, New J ersey 08801 \\ Received March 5, 1998; Revised Manuscript Received August 27, 1998
}

\begin{abstract}
We model the arm of a star polymer as an anchored random walk in an array of fixed obstacles with the added assumption of an energetic cost associated with the walk retracing its previous step. By including this energetic penalty for such "hairpin" turns on the chain, we are able to account for the thermorheological complexity of long-branched hydrogenated polybutadiene yet not introduce deviations from time-temperature superposition in a melt of the linear chains. We also show that the same assumption leads to a prediction for the thermal expansion coefficient of the linear chains that is in reasonable agreement with the latest data.
\end{abstract}

\section{Introduction}

The assumption of thermorheological simplicity is of great utility in the interpretation of rheological data. Since a polymer chain explores new conformational microstates via an activated process occurring on a given microscopic time scale, one can explore the relaxational dynamics of polymers over time scales much longer or shorter than this fundamental time by varying the temperature of the sample and thereby varying this microscopic time with respect to a given perturbation frequency or shear rate. Data taken at these various temperatures can then be related to a reference temperature through a single Arrhenius factor that accounts for changes in this fundamental time scale. ${ }^{1}$ The process of creating this master rheological curve at a single reference temperature from experiments at various temperatures is know as time-temperature superposition and thermorheological simplicity expresses the existence of these data collapse onto a master curve via a one-parameter rescaling of frequency.

The application of this sort of analysis to linear polymer melts is standard. For melts of star polymers, however, the assumption of thermorheol ogical simplicity can lead to inconsistencies in at least some chemical systems. Star polymers being the simplest type of branched polymers are model systems for a variety of materials with long-chain branching. Thus, understanding the failure of simple time-temperature superposition in some of these systems is not only interesting in its own right but also a necessary step toward the coherent interpretation of rheological data required to test more refined theories of stress relaxation in the presence of long-chain branching.

The appearance of thermorheol ogical complexity essentially depends on the chemistry of the polymer chain as well as on the presence of branches. For example, it has been found that the temperature dependence of polyisoprene stars is identical to that of linear polyisoprene. ${ }^{2,3}$ In contrast, long branches in hydrogenated polybutadiene (HPB) introduce an anomalous temperature dependence into its relaxational spectrum. Furthermore, the effect is frequency-dependent. For example, the anomal ous temperature dependence in HPB affects the long time relaxational processes more strongly than those occurring at shorter times. (In particular, this material appears to remain thermorheologically simple for frequencies near the rubber plateau with an activation energy equal to its value in a linear HPB melt. ${ }^{4,5}$ )

Star melts of HPB present other problems to the theorist. While the present theory of stress relaxation in star polymer melts ${ }^{7-11}$ has met with considerable success in elucidating both the exponential dependence of the melt viscosity upon arm molecular weight and the particular shape of star relaxational spectra, its application to melts of hydrogenated polybutadiene (HPB) reveals significant discrepancies between theory and experiment. In particular, one finds such discrepancies between the measured and calculated values of the terminal times if one insists that the entanglement molecular weight of the star arms is both identical to the entanglement molecular weight of the linear material and temperature-independent. The shape of the relaxational spectra of the HPB stars in the approach to the terminal region is still well-described by the star theory, provided one allows the entanglement molecular weight to enter as a temperature-dependent fitting parameter.

In this paper we seek to explain the thermorheological complexity of these star melts by making an assumption regarding the statistics of chain conformations within thetube. We will assume that there is an energetic cost, $\Delta$, associated with the chain making a "hairpin" turn on the scale of a single entanglement length within its tube. We will then demonstrate that such an energetic contribution to the free energy of star arms will reproduce the thermorheol ogical compl exity of the star melts and therefore the temperature dependence of the effective entanglement length that must enter the star theory to describe accurately the rheol ogical spectra. We will not, however, be able to account for the apparent doubling of the entanglement molecular weight of HPB stars compared to the value obtained from studies of the linear HPB melt.

Furthermore, our assumption of an energetic contribution to the chain free energy, in addition to the dominant entropic term, will not invalidate the reptation model for stress relaxation in the linear melt. Reptation theory for the linear chains will be unaltered by our assumption because the energetically penalized chain configurations are extremely rare events, so rare that the mean waiting time for the occurrence of a hairpin is much longer than the reptation time of the linear chain. In star polymers, however, because of the 
inhibition of reptative motion by the branch point, stress relaxation relies on extremely rare events in which the star arm retracts to within one entangl ement length of the branch point. It is in such rare, retracted chain configurations that one may expect to find highly folded states of the chain in which it executes a number of hairpin turns within its tube. The additional energetic cost of these hairpins combined with the usual exponentially large (in star arm length) entropic barrier to arm retraction will produce an effective, temperaturedependent entanglement length in the star theory and explain the failure of time-temperature superposition at low frequencies (near the terminal time) where the dominant relaxation modes involve these deep retraction of the star arms.

Such an energetic penalty will have other consequences as well. The presence of this energy, $\Delta$, affects the basic random walk statistics of the chain in which the squared radius of gyration is proportional to the degree of polymerization, $\mathrm{R}_{\mathrm{g}}{ }^{2}=\mathrm{CN}$. Because of the hairpin energy, the prefactor $c$ will be temperaturedependent. This leads to a prediction for the chain thermal expansion coefficient. The thermal expansion coefficient, $\kappa$, is defined as the logarithmic derivative of the radius of gyration with respect to temperature. It will be negative for chains having the hairpin energy since, at low temperatures where the number of hairpins is small, the chain will be elongated compared to its dimensions at higher temperatures where the equilibrium population of hairpins is larger. These chains will crumple upon heating. We expect that star materials having a temperature-dependent entanglement molecular weight and exhibiting thermorheological complexity should also have negative values for their thermal expansion coefficients. This is, in fact, the case for HPB. ${ }^{12}$ A single value of $\Delta$ should account for both the anomalous temperature dependence in the star rheology and the thermal expansion coefficient.

We do not attempt to calculate the hairpin energy on the basis of a microscopic analysis of the combinations of trans and gauche bonds that produce a hairpin. First, we assume that the penalized chain conformations are tight bends in the chain only on the scale of an entanglement length. A hairpin configuration corresponds to a variety of states at the level of individual $\mathrm{C}-\mathrm{C}$ bonds. Second, modeling a hairpin based on the rotational isometric state (RIS) theory suggests that this hairpin energy is calculable in terms of purely local chain structure. Nonlocal interactions due to the repulsi on between monomers separated by several backbone carbons which are brought close together by the hairpin bend are also responsible for the energy cost of these configurations. It should be noted that recent work by Lohse et al. ${ }^{3}$ shows that the RIS theory predicts poorly the observed thermal expansion coefficients in HPB.

The paper is organized as follows: In section 2A we introduce a lattice model for the star arm configurations which incorporates the hairpin energy. In section 2B we discuss a continuum generalization of this lattice model. Using this continuum model in section $3 \mathrm{~A}$, we determine the modified probability distribution for the distance of the arm end from its branch point. Combining this function with the star arm retraction theory, ${ }^{11}$ we then produce, in section $3 \mathrm{~B}$, the new rheological spectra. In section $3 \mathrm{C}$ we compute the radius of gyration of a linear chain having a hairpin energy to determine the thermal expansion coefficient. The reader

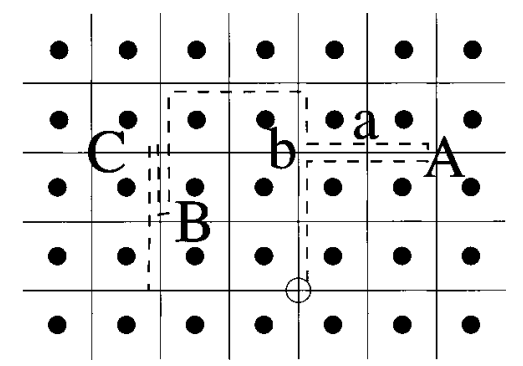

Figure 1. A two-dimensional example of a random walk on the obstacle lattice with coordination number $z=4$. The walk represented by the dashed line is anchored at the open circle. This walk has three energetically penalized hairpin turns labeled A, B, and C.

who is uninterested in the mathematical details of our work is encouraged to skip over section 3A. In section 4 we compare these predictions of the augmented star theory and the thermal expansion coefficient to the data of Raju et al. ${ }^{6}$ and Lohse et al., ${ }^{12}$ respectively. In the new theory there is indeed data collapse of rheological spectra from a number of temperatures onto a single master curve. The same value of hairpin energy, $\Delta$, which produces these data collapses also gives a chain thermal expansion coefficient for HPB which is of the correct sign but differs in magnitude from the accepted value by an order of magnitude. In section 5 we further explore the predictions of the hairpin model with particular regard to the probability of deep arm retractions and show that the probability distribution obtained in section $3 \mathrm{~A}$ is only approximately correct in the combined limit of large hairpin energy and deep arm retractions. We go on to show that additional corrections to the deep arm retraction probability do not significantly effect our earlier results. Finally, we conclude in section 6 by discussing these results and suggesting that, on the basis of the present theory, the appearance of thermorheological complexity in star polymers should correlate with negative thermal expansion coefficients of the linear chains having the same chemistry.

\section{The Model}

A. Random Walks on a Lattice. To investigate the statistical mechanics of a star arm in an entangled melt, we turn to a lattice model to enumerate the various chain configurations. We replace the topological interactions between a given polymer chain (i.e., star arm) and the surrounding melt by an array of fixed obstacles that the chain cannot penetrate. We represent a given star arm without excluded volume self-interactions as a random walk of step length a within the lattice of permanent obstacles that also has a lattice spacing of a. (Our lattice model is an extension of the model studied by Nechaev et al. ${ }^{13,14}$ in which we count the hairpin turns executed by the walk.) The coordination number of the lattice is $z$ and the random walk is anchored with one end at the origin while the other end of the chain is free to move-see Figure 1.

On the lattice we may unambiguously define these hairpin turns as instances where the consecutive bonds of the random walk representing the polymer connect the same lattice points. Now consider the set configurations of the chain that are equivalent up to a continuous deformation which does not pass the walk through an obstacle. The shortest walk in this set of topologically equivalent walks will be called the primitive path of the 


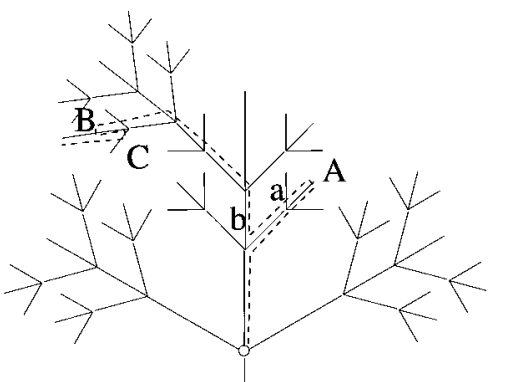

Figure 2. The random walk on a Cayley tree that corresponds to the walk in Figure 1 . The corresponding hairpins and the origin are labeled as those in Figure 1. For clarity not all branches are drawn to the same depth.

set. More physically, one may think of reeling in the excess length of the walk that is stored in unentangled loops, while keeping both the origin and ending point of the walk fixed, to produce the primitive path of a particular class of walks.

It should be noted that these walks may be put into a one-to-one correspondence with walks on a Cayley tree of the same coordination number. ${ }^{13}$ In Figure 2 we show the Cayley tree walk corresponding to the random walk of Figure 1 with equivalent points labeled.

To investigate the statistics of random walks on the Cayley tree, we define the following quantities: Let $P_{n, m}$ be the probability of finding a random walk of $n$ steps having a primitive path of $\mathrm{m}$ steps that is currently outbound. By "outbound" we mean that the direction of the last step in the walk increased the length of the primitive path by one unit. Let $\mathrm{Q}_{n, m}$ be the corresponding probability of finding an inbound walk of $n$ steps having a primitive path equal to $\mathrm{m}$ steps. Defined analogously to "outbound", "inbound" is taken to mean that the walk reduced its primitive path by one unit on the last step taken.

First, consider a random walker that is far from the origin of the Cayley tree so we may ignore the boundary conditions there for the moment. At any given step the walker has probability $p$ of taking any of $z-1$ steps that do not cause it to retrace its last step. The walker also has the probability $\mathrm{pe}^{-\Delta / \mathrm{T}}$ retracing this last step. We normalize these probabilities by setting

$$
p=\frac{1}{z-1+e^{-\Delta / T}}
$$

We now are able to derive recursion relations for $P_{n, m}$ and $Q_{n, m}$. We ask what is the probability of finding a $\mathrm{P}$-walker (outbound) with primitive path $\mathrm{m}$ and length of walk $n$. First of all, any $P_{n-1, m-1}$ walk has a $p(z-1)$ chance of taking its next step so as to increase the primitive path of the walk and thereby become a $P_{n, m}$ walk. Now, a Q-walk (inbound) with primitive path $\mathrm{m}$ - 1 and length $n-1$ can make a hairpin turn to become a $P_{n, m}$ with probability equal to $\mathrm{pe}^{-\Delta / T}$. An example of this transition from a Q- to $\mathrm{P}$-walk is shown in Figures 1 and 2 at hairpin C. However, a Q-walk may also become a P-walk without making a hairpin turn, simply by selecting one of the $z-2$ directions that head toward neither the origin nor the previous step of the walk. This second possi bility is demonstrated at point b in Figures 1 and 2 where the Q-walk becomes a P-walk. Thus, the total probability that a $Q_{n-1, m-1}$-walk becomes a $P_{n, m^{-}}$ walk is the sum, $p\left(z-2+\mathrm{e}^{-\Delta / T}\right)$, of the probabilities of the two possible $\mathrm{Q} \rightarrow \mathrm{P}$ transitions. Collecting the above remarks, we may write the following recursion relation:

$$
\begin{array}{r}
P_{n, m}=p(z-1) P_{n-1, m-1}+p\left(z-2+e^{-\Delta / T}\right) Q_{n-1, m-1} \\
\text { for } m \geq 2
\end{array}
$$

The condition requiring the walker to be at least two steps from the origin is necessary to account for the boundary conditions that will be imposed there. We return to this point after deriving the recursion relation for Q-walks that are not too close to the origin.

Consider the probability of finding a Q-walk with primitive path $\mathrm{m}$ and length $\mathrm{n}$. With probability $\mathrm{p}, \mathrm{a}$ $\mathrm{Q}_{\mathrm{n}-1, \mathrm{~m}+1}$ remains a Q-walk on the next step by continuing toward the origin and becoming a $\mathrm{Q}_{n, m}$-walk. This case is illustrated at point a in Figures 1 and 2 . There is also the probability pe ${ }^{-\Delta / T}$ that a $P_{n-1, m+1}$-walk makes a hairpin turn to to become a $Q_{n, m}$ on the subsequent step as shown at points A and B in Figures 1 and 2 . Collecting these terms, we write the second recursion relation:

$$
Q_{n, m}=p Q_{n-1, m+1}+p e^{-\Delta / T} P_{n-1, m+1} \quad \text { for } m \geq 1
$$

To complete the set of recursion relations, we must augment eqs 2 and 3 with boundary conditions at the origin and an initial condition. First, we consider the boundary conditions at the origin.

We observe that all walks that are currently at the origin are, by definition, P-walks since any of the z-steps available to the walker increase the primitive path of the walk from zero to one. Similarly, it is clear that the probability of finding a P-walker one step away from the origin at "time" $n$ (We may equivalently consider the first index as a time, counting the number of steps that the walker had taken since its introduction into the lattice. This is clearly consistent with the original definition of the $n$ as the number of steps in the random walk.) is equal to the probability of finding a P-walker at the origin on the previous time step, $\mathrm{n}-1$. On the basis of these considerations, we can complete the recursion relations with the following boundary conditions:

$$
\begin{gathered}
Q_{n, 0}=0 \\
P_{n, 1}=P_{n-1,0} \\
P_{n, 0}=p e^{-\Delta / T} P_{n-1,1}+p Q_{n-1,1}
\end{gathered}
$$

Equation 4 simply notes that there are no Q-walkers at the origin and eq 5 shows that all walks at the origin will be outbound and one step away after the next move. In eq 6 the first term accounts for outbound, P-walkers that make a hairpin to return to the origin, yet because of the special nature of the origin, they remain outbound, P-walkers. The second term represents the probability of a inbound, Q-walker reaching the origin and thereby becoming a P-walker. Last, the initial condition is that we introduce a walker at the origin so

$$
\mathrm{P}_{0, \mathrm{~m}}=\delta_{0, \mathrm{~m}}
$$

It may be noted that our choice of $p$ along with the boundary conditions enforced at the origin ensures that the combined sum over the all-primitive path lengths $\mathrm{m}$ and both types of walks (P and Q) is independent of 
the number of steps or the "time". In other words, the system conserves the total number of walkers even though P- and Q-walkers can be interconverted at each step. See Appendix A for a detailed verification of the conservation of probability by the recursion relations. In the limit that the hairpin energy is 0 the recursion relations, along with the boundary and initial conditions for the combined distribution of $\mathrm{P}$ and $\mathrm{Q}$, walks reduce to the model studied by Nechaev et al.

B. The Continuum Limit. To reexamine the theory of stress relaxation in stars, we need to find the probability of arm retractions that bring the tip of the arm to a distance x away from the branch point. In the limit that the star arm is long we may determine this probability using the continuum limit of the recursion relations introduced above. To that end we introduce the continuous variables, $x$ and $t$, equal to the length of the primitive path of the chain and the total length of the chain, respectively. In this section we show that the probability distribution of walkers in the continuum limit can be obtained as the solution of a one-dimensional advection-diffusion equation where the primitive path length, $x$, plays the role of space and the chain length, $t$, plays the role of time. We will be especially interested in the retractions of long arms that bring the tip very near the branch point, so we will consider specifically the limits in which $x \ll \sqrt{t}, t \gg 1$ when studying the solution to this problem.

We now find the continuum limit of the recursion relations for $\mathrm{P}$ and $\mathrm{Q}$ far away from the origin. Working within the continuum approximation, we will later determine boundary conditions for these fields at the origin based on the requirement of the conservation of probability. In considering the system defined by the recursion relations eqs 2 and 3 , we note that there are two types of dynamics occurring on different timescales. At each time step, Q- and P-walkers are interconverted and all P-walkers step away from the origin while all Q-walkers step toward the origin. After many steps a given walker will have been interconverted between being a P-and a Q-walker many times. After many interconversions the walker will have executed a random walk in the one-dimensional space measuring its distance to the origin of the Cayley tree. It is important to note that the two subpopulations of P- and Q-walkers considered independently do not spread diffusively. The effective diffusion of the total population of walkers is achieved only after numerous interconversion events. Thus, it is reasonable to expect to find two time scales in the dynamics: a fast time scale on which the subpopulations of $\mathrm{P}$ - and Q-walkers equilibrate and a slow time scale over which the total population of the walkers diffuses.

Therefore, intuitively one expects that the time (meaning $\mathrm{n}$ ) evolution of the probability density of all walkers in this model simply diffuses about a mean that has a net velocity toward increasing $x$. This velocity will be related to the difference between the forward and backward conversion rates of P-walkers into Qwalkers. Since the rate for $\mathrm{Q} \rightarrow \mathrm{P}$ is greater than that of $P \rightarrow Q$ we expect the equilibrated population of P-walkers to be larger than that of Q-walkers so that, counting all walkers, there will be a net flow of walkers away from the origin. On the basis of this consideration, we expect that the velocity of the mean, because of the imbalance of the two populations of walkers, will at times longer than the equilibration time of the $\mathrm{P}$ - and
Q-walker populations become time-independent. Thus, the mean position of the total walker population will increase linearly in time. This linear increase complicates the transition to the continuum limit at large times since the mean of the distribution is, itself, diverging linearly in time. This complication can, of course, be accounted for by working in a frame comoving with the mean as we do below.

\section{Consequences of the Hairpin Energy}

A. Solution of the Continuum Model. We rewrite eqs 2 and 3 as

$$
\begin{aligned}
& \mathrm{P}_{\mathrm{n}, \mathrm{m}}=(1-\beta) \mathrm{P}_{\mathrm{n}-1, \mathrm{~m}-1}+\alpha \mathrm{Q}_{\mathrm{n}-1, \mathrm{~m}-1} \\
& \mathrm{Q}_{\mathrm{n}, \mathrm{m}}=(1-\alpha) \mathrm{Q}_{\mathrm{n}-1, \mathrm{~m}+1}+\beta \mathrm{P}_{\mathrm{n}-1, \mathrm{~m}+1}
\end{aligned}
$$

where we have introduced

$$
\begin{aligned}
& \alpha=\frac{z-2+e^{-\Delta / T}}{z-1+e^{-\Delta / T}} \\
& \beta=\frac{e^{-\Delta / T}}{z-1+e^{-\Delta / T}}
\end{aligned}
$$

We now explicitly define the continuous space and time variables, $(x, t)$ respectively, through $n=k^{2} t$ and $m=k x+\mu k^{2} t$. Here, $k$ is a scale factor that we will take large so that, for finite $\mathrm{x}$ and $\mathrm{t}, \mathrm{m}$ and $\mathrm{n}$ both become large. The second equation relating $\mathrm{m}$ to $\mathrm{x}$ and $t$ includes a transition to a frame moving to the right with speed $\mu$. This speed will be adjusted to allow for a sensible continuum limit. The difference in the powers of $k$ multiplying $x$ and $t$ suggest the expected diffusive scaling and is also required for the existence of the continuum limit.

Writing the continuous probability density $\mathrm{P}(\mathrm{x}, \mathrm{t})$ as

$$
P(x, t)=P_{k 2 t, k x+\mu k 2 t}
$$

and similarly for $\mathrm{Q}(\mathrm{x}, \mathrm{t})$, we may rewrite eqs 8 and 9 as

$$
\begin{array}{r}
\mathrm{P}(\mathrm{x}, \mathrm{t})=(1-\beta) \mathrm{P}\left(\mathrm{x}+\frac{\mu-1}{\mathrm{k}}, \mathrm{t}-1 / \mathrm{k}^{2}\right)+ \\
\alpha \mathrm{Q}\left(\mathrm{x}+\frac{\mu-1}{\mathrm{k}}, \mathrm{t}-1 / \mathrm{k}^{2}\right) \\
\mathrm{Q}(\mathrm{x}, \mathrm{t})=(1-\alpha) \mathrm{Q}\left(\mathrm{x}+\frac{\mu+1}{\mathrm{k}}, \mathrm{t}-1 / \mathrm{k}^{2}\right)+ \\
\beta \mathrm{P}\left(\mathrm{x}+\frac{\mu+1}{\mathrm{k}}, \mathrm{t}-1 / \mathrm{k}^{2}\right)
\end{array}
$$

We now expand the field $\mathrm{P}(\mathrm{x}, \mathrm{t})$ order by order in $\mathrm{k}$,

$$
\mathrm{P}(\mathrm{x}, \mathrm{t})=\mathrm{P}_{0}(\mathrm{x}, \mathrm{t})+\frac{1}{\mathrm{k}} \mathrm{P}_{1}(\mathrm{x}, \mathrm{t})+\frac{1}{\mathrm{k}^{2}} \mathrm{P}_{2}(\mathrm{x}, \mathrm{t})+\cdots
$$

and, of course, similarly for $\mathrm{Q}(\mathrm{x}, \mathrm{t})$. To determine the continuum limit, we simply expand for large $k$ and collect terms.

To order $k^{0}$, we note that both eqs 13 and 14 give

$$
\alpha \mathrm{Q}_{0}(\mathrm{x}, \mathrm{t})=\beta \mathrm{P}_{0}(\mathrm{x}, \mathrm{t})
$$

which, as mentioned above, expresses the fact that the rati o of the probability densities of the P- and Q-walkers is a consequence of the detailed balance of the inter- 
conversion rates. One may note that the interconversion rate from Q-walks to P-walks is given by $\alpha$ and the rate from $\mathrm{P}$ - to Q-walks is given by $\beta$-see eqs 8 and 9 . Continuing the expansion to first order in $1 / k$, we find that the sum of eqs 13 and 14 may be written as

$$
\frac{\partial \mathrm{P}_{0}}{\partial \mathbf{x}}[\mu(\alpha+\beta)-(\alpha-\beta)]=0
$$

where we have eliminated $\partial_{x} Q_{0}$ by taking the spatial derivative of eq 16 . From eq 17 we may evaluate the speed $\mu$ of the following mean:

$$
\mu(\mathrm{z}, \Delta / T)=\frac{\alpha-\beta}{\alpha+\beta}=\frac{\mathrm{z}-2}{\mathrm{z}-2+2 \mathrm{e}^{-\Delta / T}}
$$

In second equality we have used the values of the interconversion rates expressed in eqs 10 and 11 . It is a useful check to note that the mean speed would go to zero if the interconversion rates were equal. This, however, is not possible in our model with a coordination number greater than 2 . It is also worthwhile to note the speed of the mean in two limits. In the limit that $\Delta / T \rightarrow 0$ so that hairpins carry no energetic penalty, the speed of the mean becomes $(z-2) / z=+1 /(z-1) / z+$ $-1 \times 1 / z$. The speed is given by the consideration that there are $z-1$ ways that a walker can choose a direction to increase its distance from the origin by one unit and one way that it can choose a direction to decrease that distance by one unit. If $\Delta / T=0$ each choice has the probability $1 / z$. In the limit that $\Delta / T \rightarrow$ $\infty$ the walker is not allowed to make hairpin turns and the speed is simply one, since at each step a walk must increase its distance from the origin by one unit.

Using the above result for the speed of the mean, we find the first order in the 1/k equation from the recursion relations:

$$
\alpha \mathrm{Q}_{1}(\mathrm{x}, \mathrm{t})-\beta \mathrm{P}_{1}(\mathrm{x}, \mathrm{t})=\frac{2 \beta}{\alpha+\beta} \frac{\partial \mathrm{P}_{0}}{\partial \mathrm{x}}
$$

The first-order correction to the difference in the subpopulation densities is given locally by the gradient of the zeroth-order distribution. We can interpret this equation as follows: If at a point $x_{0}$ there is a positive gradient in P-walkers, then we should expect excess Q-walkers to be created to the right of $x_{0}$, but these Q-walkers, being leftward movers will contribute to an excess density of Q-walkers (above the equilibrium value) at $x_{0}$.

To find a diffusion equation for the total probability distribution, we must go to second order in $\mathrm{k}$ since second spatial derivatives and first time derivatives make their appearance at this order. After some algebra and upon transformation back to a stationary frame, we find an advection-diffusion equation for $\mathrm{P}_{0^{-}}$ $(\mathrm{x}, \mathrm{t})$ which takes the foll owing form:

$$
\frac{\partial \mathrm{P}_{0}}{\partial \mathrm{t}}=\frac{1}{2} \mathrm{D} \frac{\partial^{2} \mathrm{P}_{0}}{\partial \mathrm{x}^{2}}-\mu \frac{\partial \mathrm{P}_{0}}{\partial \mathrm{x}}
$$

where the diffusion constant is related to the interconversion rates by

$$
\mathrm{D}=\frac{4 \alpha \beta[2-\alpha-\beta]}{(\alpha+\beta)^{3}}=4 \mathrm{ze}-\Delta / \frac{\mathrm{z}-2+\mathrm{e}^{-\Delta / T}}{\left(\mathrm{z}-2+2 \mathrm{e}^{-\Delta / T}\right)^{3}}
$$

Once again, in the second equality we have recalled the values of the transition rates $\alpha$ and $\beta$ from eqs 2 and 3 . The diffusion constant goes to zero exponentially with increasing $\Delta$ as may be expected since it is only the presence of hairpins that allows the walker to return toward the origin. In the limit $\Delta \rightarrow 0$ the diffusion constant takes the following form: $4(z-1) / z^{2}$. For a trivial Cayley tree with a coordination number equal to 2 the diffusion constant takes its maximal value of 1. At a higher coordination number the ratio of the probability of taking a step toward the origin to taking a step away from the origin goes to zero as $1 / z$ so the diffusion constant goes to zero with an increasing coordination number in the same way.

To complete the description of the continuum model, we must include boundary and initial conditions. Our choice of boundary conditions is motivated by the necessity of conserving probability at the origin. Thus, at $\mathrm{x}=0$ we require the probability current given in eq 20 to be zero:

$$
\mu \mathrm{P}(\mathrm{x}=0, \mathrm{t})-\left.\frac{1}{2} \mathrm{D} \frac{\partial \mathrm{P}}{\partial \mathrm{x}}\right|_{\mathrm{x}=0}=0
$$

We also require as a boundary condition at $x \rightarrow \infty$ that $\mathrm{P}$ go to zero. Finally, as an initial condition we want to introduce a walker at the origin by setting

$$
\mathrm{P}(\mathrm{x}, \mathrm{t}=0)=\delta(\mathrm{x}-\epsilon)
$$

where we will take the limit $\epsilon \rightarrow 0^{+}$.

Working in dimensionless units we Laplace transform the field $\mathrm{P}$ :

$$
\tilde{P}(x, s)=\int_{0}^{\infty} e^{-t s} P(x, t) d t
$$

to rewrite the differential equation eq 20 as

$$
\frac{\partial^{2} \tilde{P}}{\partial x^{2}}-\frac{\partial \tilde{P}}{\partial x}-s \tilde{P}=0
$$

with the condition that

$$
\frac{\partial \tilde{P}}{\partial \mathbf{x}}\left(\mathbf{x} \rightarrow \epsilon^{+}\right)-\frac{\partial \tilde{P}}{\partial \mathbf{x}}\left(\mathbf{x} \rightarrow \epsilon^{-}\right)=-1
$$

coming from the $\partial$-function initial condition. It is clear that solutions to eq 25 take the form $\tilde{\mathrm{P}} \sim \mathrm{e}^{\wedge \times}$ where the two solutions for $\kappa$ are

$$
\kappa_{+,-}(\mathrm{s})=\frac{1 \pm \sqrt{1+4 \mathrm{~s}}}{2}
$$

with $\operatorname{Re}\left(\kappa_{+}\right)>0>\operatorname{Re}\left(\kappa_{-}\right)$for $\operatorname{Re}(\mathrm{s})>-1 / 4$. Demanding that the solution be continuous at $\mathrm{x}=\epsilon$ with $\mathrm{a}$ discontinuity in the slope prescribed by eq 26 as well as satisfying the boundary conditions at $x=0$ and $x=$ $\infty$ provides enough equations to completely determine the solution:

$$
\tilde{\mathrm{P}}(\mathrm{x}, \mathrm{s})=\frac{1}{\kappa_{+}(\mathrm{s})} \mathrm{e}^{\kappa_{-}(\mathrm{s}) \mathrm{x}}
$$

To solve the initial value problem, we must invert the Laplace transform:

$$
\mathrm{P}(\mathrm{x}, \mathrm{t})=\frac{1}{2 \pi \mathrm{i}} \int_{\mathrm{c}-\infty}^{\mathrm{c}+\infty} \frac{1}{\kappa_{+}(\mathrm{s})} \mathrm{e}^{\kappa_{-}(\mathrm{s}) \mathrm{x}+\mathrm{st}} \mathrm{ds}
$$


where $c$, the real part of $s$ on the contour of integration, is chosen so that all nonanalyticities of the integrand lie to the left of the contour.

The integrand has a branch point at $s=-1 / 4$. We change variables to move the branch point to the origin (letting $w=1+4 s$ ) and take the branch cut to lie on the negative real axis. By closing the contour so that it runs above and below the branch cut from 0 to $-\infty$, the function $P(x, t)$ can be written as an integral involving the discontinuity across the branch cut:

$$
P(x, t)=\frac{e^{(2 x-t) / 4}}{2 \pi}\left\{\left.\right|_{A}+I_{B}\right\}
$$

where the integrals I ${ }_{A}, I_{B}$ are given by

$$
\begin{gathered}
\mathrm{I}_{A}=\int_{0}^{\infty} d p \frac{e^{-p t / 4}}{1+p} \sin \left(\frac{x \sqrt{p}}{2}\right) \\
I_{B}=\int_{0}^{\infty} d p \frac{e^{-p t / 4}}{1+p} \sqrt{p} \cos \left(\frac{x \sqrt{p}}{2}\right)
\end{gathered}
$$

The remaining integrals we approximate for the limit of long times, or in other words, for long polymer chains. The first integral can be rewritten as

$$
I_{A}=\frac{4}{t} e^{-x^{2} / 4 t} \operatorname{lm} \int_{-\infty}^{\infty} d z \frac{e^{-z^{2}}\left(z+i \frac{x}{2 \sqrt{t}}\right)}{1+\frac{4}{t}\left(z+i \frac{x}{2 \sqrt{t}}\right)^{2}}
$$

and similarly for the second integral

$$
I_{B}=\frac{8}{t^{3 / 2}} e^{-x^{2} / 4 t} \operatorname{Re} \int_{-\infty}^{\infty} d z \frac{e^{-z^{2}}\left(z+i \frac{x}{2 \sqrt{t}}\right)^{2}}{1+\frac{4}{t}\left(z+i \frac{x}{2 \sqrt{t}}\right)^{2}}
$$

We now expand the above integrands assuming that $x$ is at most on the order of $\sqrt{t}$ and that $t$ is much greater than $1: \mathrm{x} \sim \sqrt{\mathrm{t}} \ll \mathrm{t}, \mathrm{t} \gg 1$. Recall that we want to use the above probability distribution to discuss star arm retraction events for which these limits are applicable. We then may estimate the integrals eqs 33 and 34 as

$$
\begin{aligned}
& \mathrm{I}_{A} \simeq \frac{2 \sqrt{\pi} x}{t^{3 / 2}} e^{-x^{2} / 4 t}\left[1+0\left(t^{-1}\right)\right] \\
& I_{B} \simeq \frac{4 \sqrt{\pi}}{t^{3 / 2}} e^{-x^{2} / 4 t}\left[1+0\left(t^{-1}\right)\right]
\end{aligned}
$$

which leads to a result for the probability distribution in the limit that $x \sim \sqrt{t} \gg 1$. Once we have put back the dimensional variables:

$$
\begin{aligned}
& x \rightarrow 2 \frac{\mu}{D} x \\
& t \rightarrow 2 \frac{\mu^{2}}{D} t
\end{aligned}
$$

we find

$$
P(x, t) \simeq P_{0} x t^{-3 / 2} e^{-\left[(x-\mu t)^{2}\right] / 2 D t}
$$

The constants $\mu$ and $\mathrm{D}$ are given by eqs 18 and 21 , respectively. A result similar in form to eq 39 has been found for the model with $\Delta=0$ by Yurasova et al; 16 however, we disagree with their calculation of the $D$.

B. Stress Relaxation in a Star Melt. We now combine the results of the previous analysis with the existing arm retraction theory for star melts. To begin, we note that star arms may be thought of as extended in their tubes due to an entropically generated thermal tension. ${ }^{17}$ Arm retraction events within the tube occur via an activated process by which the arm climbs the entropically generated barrier, moving against the thermal tension. The probability of such a retraction event is exactly what we have calculated above. By writing that probability in a form suggestive of an activated process by setting the probability of a retraction back to $x$ equal to the exponentiated free energy change of that event divided by $\mathrm{k}_{\mathrm{B}} \mathrm{T}$ :

$$
\mathrm{P}(\mathrm{x})=\mathrm{P}_{0} \mathrm{e}^{-\mathrm{U}(\mathrm{x}) / \mathrm{K}_{\mathrm{B}} T}
$$

We find the effective potential, $\mathrm{U}(\mathrm{x})$, for an arm retraction to $x$. As seen from eqs 39 and 40, the potential is quadratic up to logarithmic corrections. We change variables from $x$ to $s$, which measures the fractional change in the primitive path of the star arm relative to its equilibrium primitive path length; $S=(\bar{L}-L) / \bar{L}$ where $\bar{L}$ is the equilibrium length of the primitive path of the star arm. When the arm is at its equilibrium length, $s=0$. At full retraction to the branch point, $s$ $=1$. We find (setting $k_{B} T=1$ )

$$
\mathrm{U}(\mathrm{s})=\frac{\mu(\mathrm{z}, \Delta / \mathrm{T})}{2 \mathrm{D}(\mathrm{z}, \Delta / \mathrm{T})} \frac{\mathrm{N}}{\mathrm{N}_{\mathrm{e}}} \mathrm{s}^{2}
$$

In this expression for the effective arm potential there is still a dependence on $z$, the coordination number of thelattice, which is a necessary but artificial feature of our model. We may choose this value by requiring that, in the event that $\Delta=0$, we return to the standard star relaxation model where the numerical value of the prefactor is equal to the off-lattice "tube model" value of $15 / 8.11$ This correspondence with the standard model sets $z=16.07$ (which is at least a reasonable size, if not an integer). For general values of $\Delta$ we may rewrite the above result, eq 41, in the form

$$
U(s)=\frac{15}{8} \frac{N}{N_{e}^{e f f}(\Delta / T)} s^{2}
$$

where we have introduced an effective entanglement length for the star arm that is now temperature dependent:

$$
\frac{N_{\mathrm{e}}^{\mathrm{eff}}(\Delta / T)}{\mathrm{N}_{\mathrm{e}}} \frac{15 z \mathrm{e}^{-\Delta / T}}{\mathrm{z}-2} \frac{\mathrm{z}-2+\mathrm{e}^{-\Delta / T}}{\left(\mathrm{z}-2+2 \mathrm{e}^{-\Delta / T}\right)^{2}}
$$

where $z$ has al ready been determined above.

Examining eq 42, we see that changes to the star arm retraction theory due to the penalized hairpins are confined to a reinterpretation of a single parameter, the arm entanglement length. It is through the temperature dependence of this parameter that the thermorheological complexity of these materials arises. To complete this section, we sketch the basics of the star arm retraction theory. 
When a melt of star polymers is subjected to a step strain, within linear response, the star arms store stress through their loss of orientational isotropy. The star molecules relax stress and recover their isotropy via arm retraction events in which the arms retract in their tubes a fractional distance $s$ and then randomly extend back into the melt. The stress stored in the melt as a function of time is then proportional to the mean fraction of the arms that have not yet retracted. To determine this stress relaxation function, we must calculate the first passage of time for the arm tip at a point s down the tube. Clearly, this time can be written as

$$
\tau(\mathrm{s})=\hat{\tau} \mathrm{e}^{\mathrm{U}(\mathrm{s})}
$$

where $\hat{\tau}$ is the inverse attempt frequency. To determine the attempt frequency, we note from Helfand et al. ${ }^{9}$ that there is an exponential separation of first passage times with increasing s so we may take the time to reach $s$ as the mean time between attempts of the arm to reach $\mathrm{s}$ $+\delta$ s. We can write

$$
\ln \tau(\mathrm{s}+\delta \mathrm{s})-\ln \tau(\mathrm{s})=\mathrm{U}(\mathrm{s}+\delta \mathrm{s})-\mathrm{U}(\mathrm{s})
$$

or, taking the limit $\delta s \rightarrow 0$

$$
\frac{\mathrm{d}}{\mathrm{ds}} \ln \tau(\mathrm{s})=\frac{\mathrm{d}}{\mathrm{ds}} \mathrm{U}(\mathrm{s})
$$

The differential equation, eq 46 , as it stands, does produce the observed exponential dependence of both the terminal time $\tau(\mathrm{s}=1)=\tau_{\text {term }}$ and the viscosity (via $\eta$ $\left.\sim \mathrm{G}_{0} \tau_{\text {term }}\right)$ on the length of the star arms but predicts too strong an exponential dependence on this quantity.

This shortcoming can be corrected in large part by the dynamic dilution hypothesis first presented by Ball and McLeish. ${ }^{10}$ They assumed that only part of the melt is effective in entangling the retracting star arms. In particular, only that fraction of the melt made up of unrelaxed star arms is effective at producing entanglements over times comparable to the arm retraction times that further relax stress. To see this point, consider a relaxed section of arm (arm 1) a fractional distance $s_{1}$ from its tip. If it is presently helping to define the confining tube of another arm (arm 2) at a spot $s_{2}, s_{2}>s_{1}$ on the tube of arm 2, the relaxed section of arm 1 will retract back to $s_{1}$ many times during the mean first passage of time at $s_{2}$ of the arm 2 and thus be ineffective in producing entanglements on the time scale of present interest. The entanglement network is diluted by a factor equal to the volume fraction of relaxed arms, $\phi$, so the effective entanglement length becomes s-dependent

$$
\mathrm{N}_{\mathrm{e}}^{\mathrm{eff}}(\mathrm{S} ; \Delta / T)=\frac{\mathrm{N}_{\mathrm{e}}^{\mathrm{eff}}(\Delta / T)}{\phi}=\frac{\mathrm{N}_{\mathrm{e}}^{\mathrm{eff}}(\Delta / \mathrm{T})}{1-\mathrm{S}}
$$

where 1 - $s$ counts the unrelaxed fraction of the melt.

Using the dynamic dilution hypothesis, we multiply the right-hand side of eq 46 by ( $1-\mathrm{s}$ ) and upon integrating we find the solution of this differential equation to be

$$
\tau(\mathrm{s})=\tau_{\mathrm{e}} \exp \left[\frac{15 \mathrm{~N}}{8 \mathrm{~N}_{\mathrm{e}}^{\mathrm{eff}}(\Delta / T)}\left(s^{2}-\frac{2 \mathrm{~s}^{2}}{3}\right)\right]
$$

where $\tau_{\mathrm{e}}$, the fundamental microscopic time scale for the chain's exploration of its tube, is the Rouse time of an entanglement segment.

To complete this review of the theory, we recall the material function that we wish to calculate-the response function $\mathrm{G}(\mathrm{t})$ that relates the shear stress to the shear strain history of the melt:

$$
\sigma(\mathrm{t})=\int_{-\infty}^{\mathrm{t}} \mathrm{d \textrm {t } ^ { \prime }} \mathrm{G}\left(\mathrm{t}-\mathrm{t}^{\prime}\right) \dot{\gamma}\left(\mathrm{t}^{\prime}\right)
$$

The modulus, $\mathrm{G}(\mathrm{t})$, can be written as

$$
\mathrm{G}(\mathrm{t})=2 \mathrm{G}_{0} \int_{0}^{1} \mathrm{ds}(1-\mathrm{s}) \mathrm{e}^{\mathrm{t} / \tau(\mathrm{s})}
$$

by adding the contribution to the stress relaxation, dG, coming from arm segments within ds of $s$ that have a relaxation time of $\tau(\mathrm{s})$ and noting that the modulus is proportional to the square of the diluted, (by dynamic dilution) unrelaxed volume fraction so: $\mathrm{dG}=$ $\mathrm{d}\left(\mathrm{G}_{0} \phi^{2}(\mathrm{~s})\right) \exp (\mathrm{t} / \tau(\mathrm{s})) .11,18$ In what follows we will be interested in the stress relaxation function in frequency space obtained by the standard Fourier transform. ${ }^{17}$

Last, we comment that our modified star theory incorporates two of the refinements first introduced by Milner and McLeish. ${ }^{11}$ The activated retraction theory overestimates the early relaxation times in which the star arms retract less than $\mathrm{k}_{\mathrm{B}} \mathrm{T}$ up the barrier. The chain tip in this early stage of stress relaxation is able to retract by making Rouse moves and, in effect, is unaware of its connection to the branch point. A theory with the correct early-time stress relaxation via Rouse modes that crosses over to activated retraction has been shown to fit well star rheological spectra by correcting previous discrepancies at high frequencies. ${ }^{11}$ This theory also includes in the expression for the rel axation time $\tau(\mathrm{s})$ a preexponential factor that is computed from a direct evaluation of the first passage of time of the star arm tip a fractional distance s down its tube. ${ }^{11}$ We also include this second refinement to the star theory but do not include, in the present work, the Colby, Rubenstein scaling for the entanglement length versus the volume fraction-a third refinement introduced by Milner and McLeish. ${ }^{11}$

C. Thermal Expansion of Linear Chains. In this section we consider the thermal derivative of the radius of gyration of a linear chain having the hairpin energy. Without the hairpin turn energy, $\Delta$, the free energy of the chain would be of purely entropic origin and thus be proportional to $k_{B} T$. The free energy minimum would therefore be independent of temperature and we would find that the thermal expansion coefficient of the chain, $\kappa$, vanishes. The inclusion of the hairpin energy will produce a negative value of $\kappa$ since raising the temperature will increase the equilibrium number of hairpins and thereby decrease the radius of gyration of the chain. To see this quantitatively, we need to find the mean value of the magnitude of the end-to-end vector of the chain.

Let each segment of the chain be represented by the vector $\sigma_{i}$ where the index $\mathrm{i}$, counting the segments of the chain runs from 1 to $N$. Since we are interested only in the logarithmic derivative of the radius of gyration, we choose, without loss of generality, the 
length of each segment to be $1:|\sigma|=1$. The quantity we are interested in is then

$$
\left\langle\mathrm{R}^{2}\right\rangle=\sum_{\mathrm{i}, \mathrm{j}=1}^{\mathrm{N}}\left\langle\sigma_{\mathrm{i}} \cdot \sigma_{\mathrm{j}}\right\rangle=\mathrm{N}+\frac{1}{2} \sum_{\mathrm{i}<\mathrm{j}}^{\mathrm{N}}\left\langle\sigma_{\mathrm{j}} \cdot \sigma_{\mathrm{j}}\right\rangle
$$

Without the hairpin energy, the different segments are uncorrelated with each other so the last summation vanishes. The hairpin energy introduces correlations between these various vectors which we now investigate, once again, on a z-coordinated lattice.

Pick an arbitrary segment which we label the ith segment. The direction of $\sigma_{\mathrm{i}}$ will define the forward direction. We ask what is the probability that the $\sigma_{i+n}$ vector lies in the forward direction. Similarly, we will calculate the probability that that vector is antiparallel to $\sigma_{\mathrm{i}}$ which we call the backward direction. We also calculate the probability that the $\sigma_{i+n}$ vector lies in any of the $z-2$ directions that are neither parallel nor antiparallel to the forward direction. These directions will be called collectively the side directions. We collect these probabilities into a three-component vector:

$$
\vec{P}_{i+n}=\left(\begin{array}{l}
P_{\text {front }}(n) \\
P_{\text {side }}(n) \\
P_{\text {back }}(n)
\end{array}\right)
$$

From our previous analysis of the hairpin penalized walk on the lattice we know $\overrightarrow{\mathrm{P}}_{\mathrm{i}+1}$ :

$$
\vec{P}_{i+1}=p\left(\begin{array}{l}
1 \\
z-2 \\
e^{-\Delta / T}
\end{array}\right)
$$

To solve in general for the probability vector for the orientation (relative to the ith bond) of the bond vector $\mathrm{n}$ steps away from the ith one, we may derive a transfer matrix $\vec{\Psi}$. As explained in Appendix $B, \Psi$ is given by

$$
\vec{\Psi}=p\left(\begin{array}{lll}
1 & 1 & e^{-\Delta /} \\
z-2 & z-3+e^{-\Delta / T} & z-2 \\
e^{-\Delta / T} & 1 & 1
\end{array}\right)
$$

and we calculate the nth probability vector by the repeated application of the transfer matrix:

$$
\overrightarrow{\mathrm{P}}_{\mathrm{i}+\mathrm{n}}=\overleftrightarrow{\Psi}^{\mathrm{n}-1} \cdot \overrightarrow{\mathrm{P}}_{\mathrm{i}+1}
$$
are

Diagonalizing $\overleftrightarrow{\Psi}$ we find that the three eigenvalues

$$
\lambda_{1,2,3}=\left\{\frac{-\beta}{z-\beta}, \frac{\beta}{z-\beta}, 1\right\}
$$

where $\beta=1-\mathrm{e}^{-\Delta /}$. To determine $\overrightarrow{\mathrm{P}}_{\mathrm{i}+\mathrm{n}}$, we decompose the vector $\overrightarrow{\mathrm{P}}_{\mathrm{i}+1}$ into components al ong the eigenvectors corresponding to the eigenvalues shown in eq 56 , so the repeated application of $\Psi$ is trivial. Then, it is simple to show that the sought-after probabilities are given as

$$
\begin{gathered}
\mathrm{P}_{\text {front }}(\mathrm{n}+\mathrm{i})=\frac{1}{\mathrm{z}}+\frac{1}{2}\left(\frac{-\beta}{\mathrm{z}-\beta}\right)^{\mathrm{n}}\left[1+(-1)^{\mathrm{n} z-2} \mathrm{z}\right] \\
\mathrm{P}_{\text {side }}(\mathrm{n}+\mathrm{i})=\frac{\mathrm{z}-2}{\mathrm{z}}\left[1+(-1)^{\mathrm{n}}\left(\frac{\beta}{\mathrm{z}-\beta}\right)^{\mathrm{n}}\right]
\end{gathered}
$$

$$
\mathrm{P}_{\text {back }}(\mathrm{n}+\mathrm{i})=\frac{1}{\mathrm{z}}-\frac{1}{2}\left(\frac{\beta}{\mathrm{z}-\beta}\right)^{\mathrm{n}}\left[1-(-1)^{\mathrm{n}} \frac{\mathrm{z}-2}{\mathrm{z}}\right]
$$

It is important to note that the correlations introduced by the hairpin energy vanish in the limit that $\Delta=0 \Rightarrow$ $\beta=0$. In this limit all the probabilities become n-independent. The side probability is simply $z-2$ times the forward and backward ones since there are that many more directions included in the set of sideway ones. Also, for all values of $\Delta$, including the limit of forbidden hairpins, $\Delta \rightarrow \infty$, the probabilities decay toward their uncorrelated values exponentially with $\mathrm{n}$. The local correlations, however, are essential to understand the change in chain dimensions with temperature.

To complete this last step and calculate the chain dimensions, we return to eq 51 and write

$$
\begin{aligned}
\sum_{\mathrm{i}<j}^{\mathrm{N}}\left\langle\sigma_{\mathrm{i}} \cdot \sigma_{\mathrm{j}}\right\rangle & =\left\langle\sum_{\mathrm{i}=1}^{\mathrm{N}-1} \sigma_{\mathrm{i}} \cdot \sum_{\mathrm{k}=1}^{\mathrm{N}-\mathrm{i}} \sigma_{\mathrm{i}+\mathrm{k}}\right\rangle \\
& =\sum_{\mathrm{i}=1}^{\mathrm{N}-1 \mathrm{~N}-\mathrm{i}} \sum_{\mathrm{k}=1}\left[\mathrm{P}_{\text {front }}(\mathrm{i}+\mathrm{k})-\mathrm{P}_{\text {back }}(\mathrm{i}+\mathrm{k})\right]
\end{aligned}
$$

The inversion symmetry of a Bravais lattice about a lattice point ensures that the sum of lattice vectors in the "sideways" subspace vanishes.

Using the results for these probabilities in eqs 57 and 59 and doing the multiple geometric sums, we find that the radius of gyration is given by

$$
\left\langle R^{2}\right\rangle=N\left[\frac{2 z-3+3 e^{-\Delta / T}}{2 z-4+4 e^{-\Delta /}}\right]+0(1)
$$

We ignore the 0 (1) corrections since we are interested in the limit of long chains and note that we recover the basic random walk statics: $R^{2} \propto N$. There is now a temperature-dependent prefactor. The thermal expansion coefficient is then

$$
\kappa=\frac{\mathrm{d} \ln \mathrm{R}^{2}}{\mathrm{dT}}=-\frac{2 \mathrm{z} \Delta \mathrm{e}^{-\Delta / T}}{(2 \mathrm{z}-3 \beta)(2 \mathrm{z}-4 \beta) \mathrm{T}^{2}}
$$

Since we have previously determined a value for $z$, the value of $\kappa$ is fixed by the energy scale $\Delta$ that needs to be determined from star rheological data. We turn to these data next.

\section{Rheological Data}

To test the efficacy of our theory in regard to understanding the, as yet, mysterious behavior of HPB, we apply the following procedure: We take a data set from Raju ${ }^{6}$ which contains a series of HPB stars having nominal arm lengths of $13.3,16.9,20.0,37.9,39.4$, and 45.4 entanglement lengths. By "nomi nal" we mean the length of the arms as determined by taking the molecular weight of the material and dividing by the number of arms and the entanglement molecular weight, as determined by the plateau modulus of the linear chain melt having the same chemistry.

The data were taken at three temperatures: 130, 160, and $190^{\circ} \mathrm{C}$. We assume the thermorheol ogical simplicity of the data at a high frequency (which has been successfully tested befor ${ }^{4}$ ) and use time-temperature superposition to collapse the modulus versus frequency data onto a single curve at the high-frequency endwhere $\mathrm{G}$ attains the plateau modulus. We find that an 


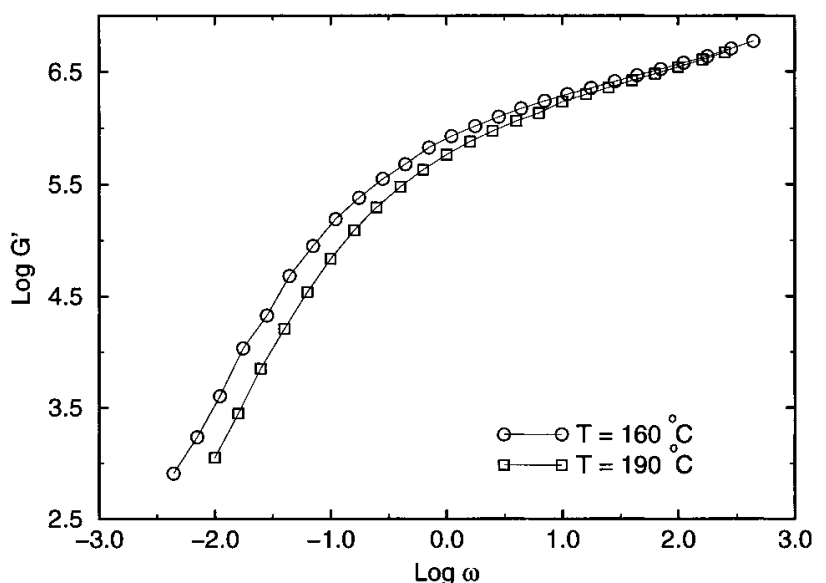

Figure 3. The storage modulus of a melt of three-arm stars whose arm molecular weight is $3.7 \times 10^{4} \mathrm{~g} / \mathrm{mol}$. The open circles represent the storage modulus at $\mathrm{T}=160^{\circ} \mathrm{C}$ and the open squares represent data taken at a temperature, $\mathrm{T}=190$ ${ }^{\circ} \mathrm{C}$. The lower temperature data have been shifted to the reference temperature of $190^{\circ} \mathrm{C}$ using an activation energy of $3800 \mathrm{~K}$. Note the failure of superposition that becomes especially apparent in the terminal region.

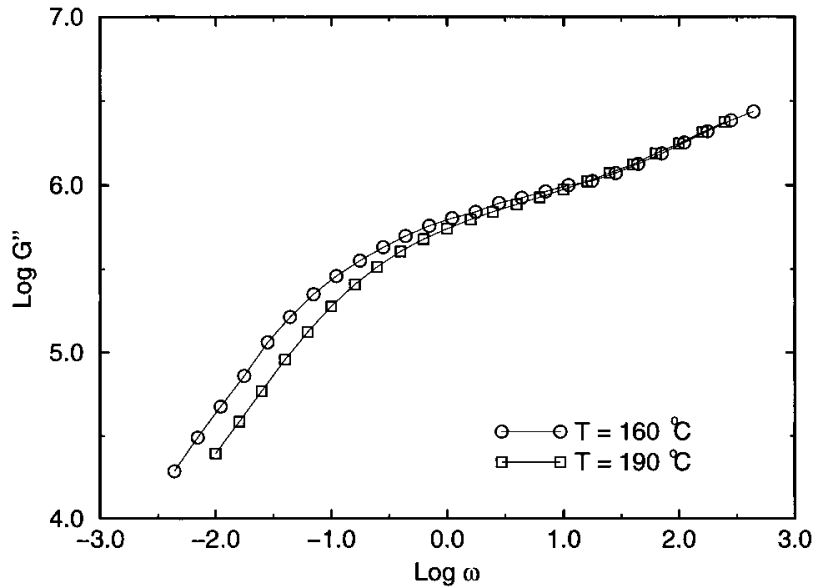

Figure 4. The loss modulus corresponding to the data shown in Figure 3.

activation energy of $3800 \mathrm{~K}$ achieves these data collapses in agreement with results from the linear melt. In Figures 3 and 4 we show the result of the timetemperature superposition with no modulus shifts of a melt of HPB three-arm stars having an arm molecular weight of $3.7 \times 10^{4} \mathrm{~g} / \mathrm{mol}$, or 37.9 entanglement lengths if we use the value of the entanglement molecular weight determined from the linear melt. ${ }^{6}$ Note that for both $\mathrm{G}^{\prime}$ and $\mathrm{G}^{\prime \prime}$ the two curves obtained at different temperatures do indeed superimpose at high frequencies but have clearly different terminal behavior.

Making such a plot for all seven data sets obtained from Raju, we attempt to fit the rheological spectra with the star theory using the entanglement molecular weight for each data set and temperature as a fitting parameter. The only other fitting parameters used are an overall scale factor for the modulus and one for time. Once chosen, the same scale factors are used in the comparison of the theory to all seven data sets.

The entanglement molecular weight is adjusted individually to fit the data for each melt and at each temperature. A representative example of such a fit is shown in Figures 5 and 6 . In Figure 5 we choose an entanglement molecular weight so that the star arms

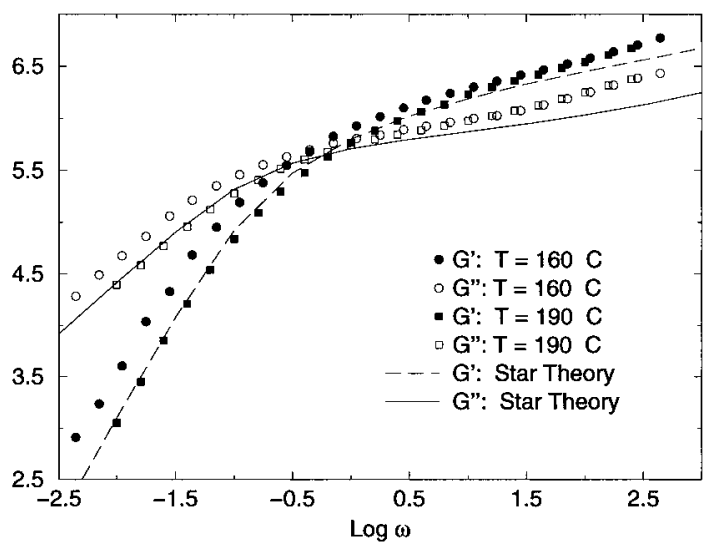

Figure 5. The rheological spectra for the same star melt as shown above in Figures 3 and 4 are now shown with fits to the star theory, taking the arm length to be 18.0 entanglement lengths. The theory with this choice of arm entanglement length fits the terminal behavior of the data taken at the higher temperature but not that taken at the lower temperature.

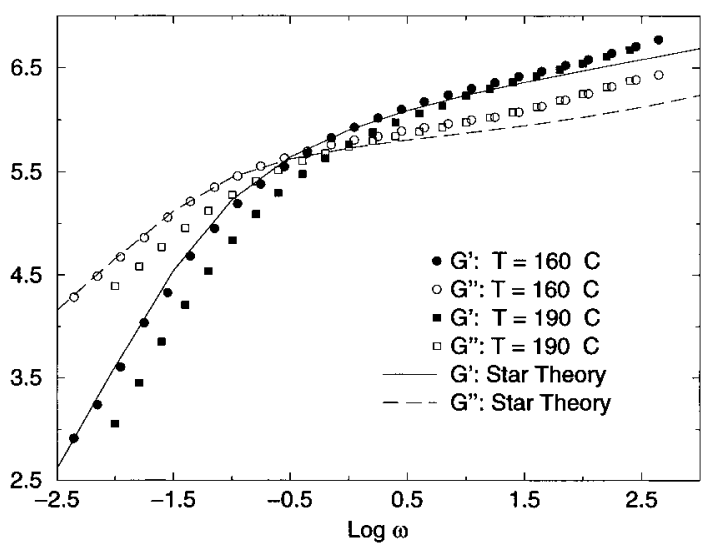

Figure 6. The rheological spectra for the same star melt as shown above in Figures 3 and 4 are now shown with fits to the star theory, taking the arm length to be 19.0 entanglement lengths. The theory with this choice of arm entanglement length fits the terminal behavior of the data taken at the lower temperature but not that taken at the higher temperature.

are 18.0 entanglement lengths long. With this choice of $\mathrm{N}_{\mathrm{e}}$ the star theory captures correctly the crossover to terminal behavior in both $\mathrm{G}^{\prime}$ and $\mathrm{G}^{\prime \prime}$ at $\mathrm{T}=190^{\circ} \mathrm{C}$. (See Figure 5.)

At the lower temperature of $\mathrm{T}=160{ }^{\circ} \mathrm{C}$ we must again adjust the length of the star arms, by changing the effective entanglement molecular weight, to bring the theory into agreement with the data. Here, we find that we must shift the effective entanglement mol ecular weight so as to make the star arms 19.0 entanglement lengths long. (See Figure 6.) Because of the exponential dependence of the terminal time on the arm length, these fits are quite precise-a change in arm length of about $1 \%$ noticeably degrades the fits to the data.

Collecting these fitting values for the various data sets, we compare the fitted length of the star arms to their nominal length. We plot the nominal value of the length of the star arms in units of entanglement segments versus the fitted value of this parameter in Figure 7. The multiple fitted values of the star arm length for a given nominal value reflect the multiple temperatures at which the melt rheology was investigated. There are two aspects of this figure worthy of note. The first is that for each nominal star arm length 


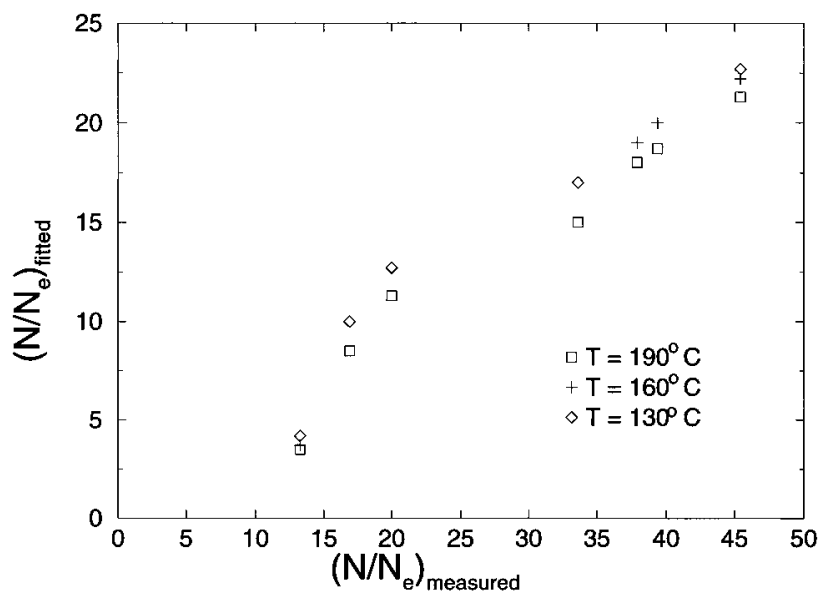

Figure 7. The graph of the nominal arm length in units of $\mathrm{Ne}$ vs the fitted value of this length. For each nominal arm length there are either two or three fitted values corresponding to the two or three temperatures at which the rheology of that particular melt was measured.

there exists a spread of fitted arm lengths in which the fitted length increases with decreasing temperature. Second, it is clear that a line drawn through the locus of points at each nominal arm length (x-axis value) does not have a slope consistent with 1 . Hence, there seems to be an overall multiplicative shift of the effective entangl ement length from linear chains to stars that is roughly independent of temperature. We return to this second point later.

On the basis of our result, eq 43, we may attempt to collapse the spread of fitting values of star arm lengths for a given nominal arm length by comparing

$$
\frac{\mathrm{N}}{\mathrm{N}_{\mathrm{e}}} \exp \left[-\Delta\left(\frac{1}{\mathrm{~T}}-\frac{1}{\mathrm{~T}_{0}}\right)\right]
$$

to the nominal star arm length. In eq 63 we once again take the reference temperature $\mathrm{T}_{0}=190{ }^{\circ} \mathrm{C}$ and choose the energy $\Delta$ as a single fitting parameter for all seven data sets. This multiplication compensates for the dominant (exponential) temperature dependence of the entanglement length and thus should lead to the collapse of the several effective entanglement lengths obtained for a single melt at several temperatures. We find that with the choice of $\Delta=380 \mathrm{~K}$ such a data collapse is possible. The resulting plot is shown in Figure 8.

The reduction of the temperature dependence of the fitting arm length is clearly apparent in all but the last data point. For this last point the shift is too large-a value of $\Delta \cong 200 \mathrm{~K}$ is required to collapse these last three data points. For this long-armed star, however, the data do not include frequencies much below the terminal time, so the fits of the star theory to this data set are most suspect.

Given this reduction of the temperature dependence of the fitting values of the star arm length, it is obvious that the fitted values of the arm lengths are close to half that of the nominal values. In other words the apparent entanglement length of the star arms is about 2.2 times that of the linear molecule. This is demonstrated in Figure 8 by the solid line which is the best linear fit to the data that goes through the origin. This line has a slope of $\sim 0.46$.

Using our temperature-dependent effective entanglement length eq 43, we are unable to account for the

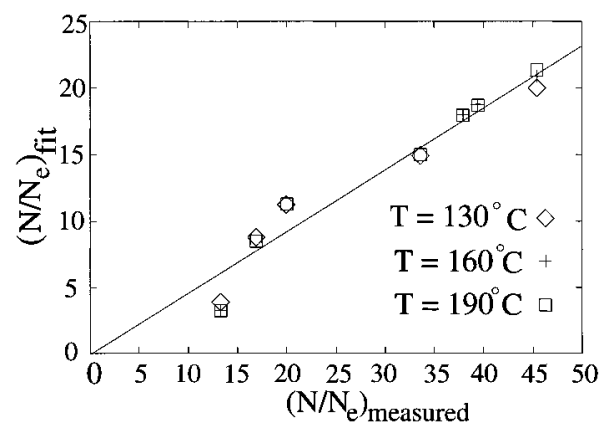

Figure 8. The graph of the nominal arm length in units of $\mathrm{N}_{\mathrm{e}}$ vs the fitted value of this length times the temperature dependent factor shown in eq 63. Whereas in Figure 7 there is a range of fitting values of the arm length for a given nominal arm length, here there is a collapse of these points. The line through the data points is the best linear fit and has a slope of $\sim 0.46$

presence of this multiplicative factor within our modified star theory. The temperature-dependent entanglement length has been designed to reach 1 in the limit that $\Delta$ goes to zero. With increasing $\Delta$ this effective entanglement length decreases monotonically.

As another consistency check on our theory, we compare the predicted value of the thermal expansion coefficient with one recently obtained from the experiment. Lohse et al. ${ }^{12}$ measured chain radii of gyration using small-angle neutron scattering for a series of poly(ethylene-1-butene) copolymers of varying ethyl branch content. They determined that the magnitude of $\kappa$ increases with an increasing ethyl branch content. For the ethyl branch content equal to that of the material of Raju, they found a thermal expansion coefficient for temperatures in the range of $300-440 \mathrm{~K}$ of $\kappa \exp =-9.5$ $\times 10^{-4} \mathrm{~K}^{-1}$ while our calculation (eq 62) produces a result of $\kappa_{\mathrm{th}}=-0.44 \times 10^{-4} \mathrm{~K}^{-1}$. The predicted chain dimension thermal expansion coefficient is of the correct sign, but the predicted effect is too small by about an order of magnitude.

The fact that our result for the thermal expansion coefficient is too small in magnitude is perhaps related to the lattice model on which it was calculated. In the lattice model, a single direction of a bond is declared a hairpin and energetically penalized, while a more realistic, continuous model would assign an energy cost to all bond angles on the unit sphere with a weighting factor that reaches a maximum for the antiparallel state. The lattice model, by including only this antiparallel state, undercounts the energy cost of most configurations and thus undercounts the temperature dependence of the radius of gyration. Neverthel ess, the rough agreement of these values with no remaining fitting parameters suggests that the notion of a hairpin energy may be more than an ad hoc fix to the star theory and, in fact, represents a useful correction to the large length scale chain dynamics coming from chain interactions on the scale of a single entanglement length.

\section{Corrections to the Quadratic Arm Potential}

In addition to the exploration of the recursion relations based on the solution of the advection-diffusion equation obtained earlier, we may directly study the recursion relations in their discrete form both numerically and analytically. The reasons for doing so are 2-fold: (i) the numerical evaluation of the recursion relations in the large $\mathrm{n}$ limit serves to verify our analytical treatment and (ii) both the numerical and 
analytic studies of the discrete recursion relations allow us to investigate regions of parameter space $(n, z$, and $\Delta$ ) in which the random walks fail to satisfy the assumptions underlying mapping of the discrete problem onto the continuum. We discuss this second reason in greater detail below.

In the following we distinguish two different analyses of the hairpin model: the study of the continuum limit of the model which yielded the advection-diffusion equation presented in section $2 \mathrm{~B}$ will be referred to as the "diffusion approximation" while the results coming from the direct investigation of the recursion relations either numerically or via generating function methods will be called the "lattice model". Of course, for the diffusion approximation to be meaningful, the results of it must approach the exact solution of the lattice model. However, in this section we specifically focus on the breakdown of the diffusion approximation and the resulting discrepancies between its predictions and those of the lattice model, which makes such a distinction necessary.

Our continuum model (diffusion approximation) for arm retraction is based on the existence of long-time diffusive dynamics of a random walker representing the tip of the retracting star arm. In the limit of large $n$ at finite $\Delta / T$ this condition is satisfied since one expects the number of hairpin turns to also be large, growing as $n \exp (-\Delta / T)$. It may be recalled that the path of a random walker will be diffusive only on time scales (i.e., numbers of steps) where the walker has made many interconversions between being a P-walk and a Q-walk. We now consider the case in which the mean number of hairpin turns on a walk is small, owing to a large hairpin energy and finite $n$. In particular, let us consider the probability of a deep retraction all the way to the branch point in this limit. In this limit the deficiencies of the diffusion approximation become most evident. From the diffusion approximation, we find that the probability of such a deep retraction is proportional to

$$
\exp \left[-\mathrm{e}^{\Delta / T}\right]
$$

There is, however, another path for the random walker which results in an unentangled return to the origin and which is more probable in the limit of large $\Delta / T$. Suppose that the walker takes $\mathrm{n} / 2$ consecutive steps as a P-walker, thus moving away from the branch point. Now the walker executes a single hairpin turn and takes $\mathrm{n} / 2$ consecutive steps as a Q-walker and so returns to the branch point. The probability of such a path is given approximately in the limit of large $\Delta$ by

$$
\frac{e^{-\Delta / T}}{(z-1)^{n / 2}}
$$

The numerator in eq 65 represents the probability of making one hairpin turn and the denominator is the probability of the returning Q-walker choosing the correct direction at each of $n / 2$ consecutive steps in order to return to the origin. It is clear that this path is highly nondiffusive and thus is not captured in the continuum, advection-diffusion analysis of the recursion relations. In addition, at fixed $\mathrm{n}$ and for $\mathrm{n} \exp (-\Delta / T)<1$, the second, nondiffusive path with probability given by eq 65 is clearly more probable than that predicted by the Gaussian model in eq 64.

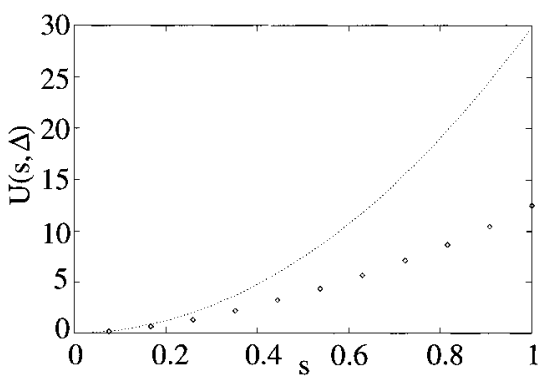

Figure 9. A comparison between the arm potential evaluated numerically directly from the recursion relations with the following parameters: $z=6, \Delta / T=1.0$, and $n=25$ (diamonds), and the best quadratic fit to the potential minimum (points). The potential, in arbitrary units, is plotted against the fractional distance that the arm has retracted, s. The best quadratic fit approaches the analytically calculated result in the limit of a large number of steps (i.e., large n). The potential determined directly from the lattice model is significantly softer for deep retractions compared to those the quadratic approximation suggests.

Although the above comparison was made in the limit of large $\Delta / T$, it provides motivation to determine the extent of the deviations of the diffusion approximation from the results of the lattice model for a less extreme choice of $\Delta / T$. To this end we examine, as an example, the recursion relations for $z=6, \Delta / T=1.0$, and $n=25$. We simply iterate the recursion relations to build up the probability distribution for the random walker and, by taking the logarithm of these probabilities, compute the effective arm potential directly. At the same time we determine the best quadratic fit to the potential minimum (at $s=0$ ). The curvature of the best quadratic fit already at 25 steps agrees closely with that curvature cal culated from the diffusion approximation. Both the best quadratic fit to the potential and the numerically determined arm potential are shown in Figure 9. It is clear from the figure that even for moderate values of $\Delta / T$ which we consider here there is a significant deviation of the arm potential from that derived by the diffusion approximation. This deviation is largest for deep retractions ( $\mathrm{s}=1$ is retraction all the way to the branch point) and, as we expected from the above argument, the actual arm potential predicted by the lattice model is softer for deep retractions than that predicted by the diffusion approximation. We have also performed an analytical calculation of the probability of deep retractions using generating functions that agrees with the numerical results and has as a limiting case for the probability of return to the origin in the large $\Delta / T$ limit the result given by eq 65 . We discuss the generating function approach in Appendix C. Finally, we re-emphasize that the magnitude of the deviation between the diffusion approximation and the lattice recursion relation is strongly $\Delta / T$-dependent. In the limit of zero $\Delta$ and $n=25$ the best quadratic fit becomes a good approximation to the lattice arm potential uniformly in $\mathrm{s}$. This best quadratic fit is also within $1 \%$ of the curvature predicted by the advectiondiffusion equation.

Given the significant and highly $\Delta$-dependent difference for the arm potential at deep retraction between the quadratic form given by the diffusion approximation and the nonquadratic form determined directly from the lattice recursion relations, it is reasonable to wonder whether the shape of the relaxational spectra themselves becomes highly $\Delta$-dependent in the hairpin model. Such a line shape dependence of the relaxational 


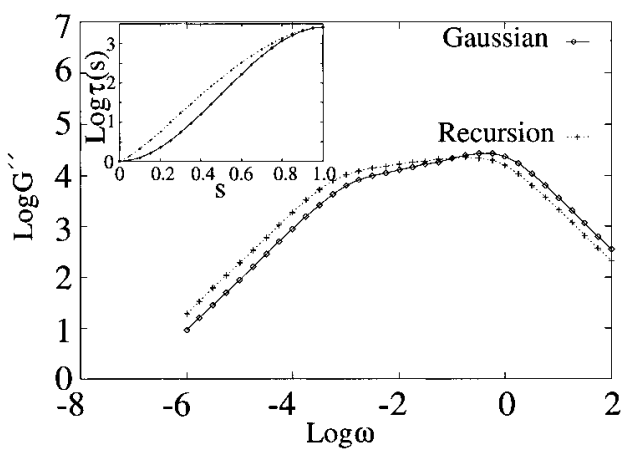

Figure 10. A comparison of the loss modulus calculated numerically from the recursion relation and the loss modulus calculated from the standard quadratic arm potential. The inset shows a comparison of the relaxation time as a function of $s$ for both the Gaussian model and the numerically evaluated hairpin model. In the lattice recursion model the parameters used are $z=16, \Delta / T=1.0$, and $n=25$. In both figures the terminal times, $\tau(\mathrm{s}=1)$, has been equated and the effects of dynamic dilution have been taken into account. Note that the shape of the relaxational spectrum does not change significantly due to the nonquadratic nature of the arm potential as calculated directly from the recursion relation. In particular, the width of the spectrum is essentially unchanged.

spectra would have severe consequences for the theory. Furthermore, the nonquadratic nature of the potential clearly calls into question the use of the diffusion approximation results in the previous section. The hallmark of the diffusion approximation is that the entire effect of the hairpin energy, $\Delta$, can be subsumed into the redefinition of a single parameter in the standard arm potential-the temperature-dependent effective entanglement length. This result ensures that there is no change in the shape of the rheol ogical spectra with changing $\Delta / T$. Recall that the experimental results suggest that changing temperature changes the effective length of the star arms but does not change the shape of the rheological spectra. Thus, if the deviation of the potential for deep retractions from the diffusion approximation results in a significant change in line shape, the hairpin model will be unable to account for this basic experimental observation.

We have computed the rheological spectra numerically directly from the lattice recursion relations by evaluating arm potential as in Figure 9 and then numerically integrating both eq 46 and the Fourier transform of eq 50 to obtain the frequency-dependent shear modulus. The result is shown in Figure 10 in which we plot the loss modulus resulting from the numerical integrations of the lattice recursion relation and that calculated using the standard quadratic arm potential. The inset of the figure shows the same comparison between the relaxation times of a segment of arm a fractional distance s from the tip. In both plots the arm length in the standard quadratic arm potential has been chosen to produce the same terminal time as the lattice recursion model in order to facilitate a comparison of the shapes of the two curves. While it is clear from the inset that the softer potential for deep retractions has an effect on the shape of $\tau(\mathrm{s})$, the line shapes of the loss moduli are clearly similar. It may be noted that the change in line shape is almost unobservable and the dominant differencelies in a small shift of the spectrum produced from the lattice model to lower frequencies.

The remarkable ineffectiveness that the difference in the deep retraction potentials has in altering the loss modulus spectrum may be traced to dynamic dilution.
First, dynamic dilution changes the trajectory of the arm tip over the potential energy landscape, $U(s, n)$, in that the effective arm length, $n$, is now a decreasing function of s. Instead of climbing the arm potential at constant $\mathrm{n}$ the arm is able to fully relax on a trajectory that never explores deep retractions at large $n$ where the softening of the arm potential is the greatest. Second, dynamic dilution introduces a reduction of the modulus scale proportional to $(1-s)^{2}$ so that the modulus is small during the period of deep retractions; thus, the final, deep retractions of the chain provide a minimal contribution to the overall modulus. Dynamic dilution validates our previous use of the diffusion approximation despite its inaccuracies with regard to deep retractions and large hairpin energies.

\section{Conclusions}

The assumption of the existence of energetically penalized chain configurations on the scale of a single entanglement length is able to simultaneously explain the thermorheological complexity of these materials that becomes apparent at low frequencies (near the terminal frequency of the melt), and produce a reasonable value for the thermal expansion coefficient of the linear chains. These results, which are based on a single fitting parameter, the energy cost of a hairpin turn in the tube, when taken together suggest that the hairpin energy is a relevant addition to the previous understanding of the relaxational dynamics of HPB. We must add that the hairpin energy assumption does nothing to resolve the puzzle of the factor of 2 discrepancy between the effective entanglement molecular weight of HPB stars and linear chains. Our model produces a monotonically decreasing effective entanglement length with increasing hairpin energy, $\Delta$, while the resolution of the difference in entangl ement lengths between the star and linear chains requires an increase in the star entanglement length with respect to that for the linear chains.

Stronger evidence for the validity of the hairpin model would come from checking the correlation between thermorheological complexity and negative chain thermal expansion coefficients. If it can be shown that thermorheological complexity in a star melt and a negative thermal expansion coefficient are commonly found together in a variety of chemical systems, then these phenomena may well be related to a single principal as this paper supposes. The experimental test of this hypothesis across a number of chemi cal systems is cl early needed. One suggestion is to consider a series of HPB polymers with a varying ethyl branch content. The data of Lohse et al. show that the thermal expansion coefficient of these materials changes their signs from negative to positive. If star polymers could be made from this series of materials, a definitive check of our present theory could be made by looking for the predicted disappearance of thermorheological complexity when $\kappa$ changes from negative to zero.

Moreover, to further check the internal consistency of our addition to the understanding of star polymer dynamics, one could study self-diffusion in star melts. Our work predicts that self-diffusion should also reveal the particular temperature dependence of the effective entangl ement length discussed above since this process also relies on arm retraction events. On the basis of the above analysis we predict an anomalous temperature dependence of the diffusion constant due to the thermally activated hairpins.

Finally, we wish to call attention to the possibility of experimentally distinguishing the hairpin model from 
the standard Gaussian theory (quadratic arm potential) of arm retraction by probing the validity of its prediction with regard to the nonquadratic nature of the arm potential for deep retractions. We have al ready noted that the predicted arm potential for deep retractions is softer than that predicted from the diffusion approximation to our theory, which itself reproduces the standard quadratic arm potential. This difference, however, is masked in the relaxational dynamics of a star melt due to the effects of dynamic dilution. The study of the diffusion of a small concentration of HPB stars in a highmolecular-weight matrix of linear HPB should bring to light the predicted nonquadratic nature of the arm potential provided that the reptation time of the linear melt is significantly longer than the terminal time of the star arms. In such circumstances the hairpin model predicts anomalously fast diffusion of the stars in the high-molecular-weight matrix compared to that predicted by the standard Gaussian arm potential.

Acknowledgment. We would like to thank Ron Larson for probing questions that stimulated this work. We also thank Dave Lohse, Ben White, and Bill Graessley for many useful discussions and V. R. Raju for providing unpublished data from his Ph.D. thesis.

\section{Appendix A: Conservation of Probability}

In this Appendix we check that probability is indeed conserved by our choice of boundary conditions. We will leave $p$ undetermined in this section and derive the choice made earlier from the conservation of the number of random walkers over time.

We begin by writing the total number of random walkers, $\mathcal{T}_{n}$, by summing over all path lengths, $m$, and over both types of walkers, $\mathrm{P}$ and Q, at a particular time n.

$$
\mathcal{T}_{\mathrm{n}}=\sum_{\mathrm{m}=0}^{\infty}\left[\mathrm{P}_{\mathrm{n}, \mathrm{m}}+\mathrm{Q}_{\mathrm{n}, \mathrm{m}}\right]
$$

Using the recursion relations, eqs 2-6, we rewrite this sum in terms of the probability distributions at the previous time step:

$$
\begin{gathered}
\mathcal{T}_{\mathrm{n}}=\sum_{\mathrm{m}=2}^{\infty}\left[(z-1) \mathrm{pP}_{\mathrm{n}-1, \mathrm{~m}-1}+(z-2+\right. \\
\left.\left.\mathrm{e}^{-\Delta / \mathrm{T}}\right) \mathrm{pQ}_{\mathrm{n}-1, \mathrm{~m}-1}\right]+\sum_{\mathrm{m}=1}^{\infty}\left(\mathrm{pQ}_{\mathrm{n}-1, \mathrm{~m}+1}+\right. \\
\left.\mathrm{pe}^{-\Delta / T} \mathrm{P}_{\mathrm{n}-1, \mathrm{~m}+1}\right)+0+\mathrm{P}_{\mathrm{n}-1,0}+\mathrm{pe}^{-\Delta / T} \mathrm{P}_{\mathrm{n}-1,1}+\mathrm{pQ}_{\mathrm{n}-1,1}
\end{gathered}
$$

Collecting terms, we can write this as

$$
\mathcal{T}_{\mathrm{n}}=\mathrm{p}\left(\mathrm{z}-1+\mathrm{e}^{-\Delta / T}\right) \mathcal{T}_{\mathrm{n}-1}
$$

which demonstrates the conservation of probability once we choose $p$ so that the prefactor of $\widetilde{T}_{n}-1$ on the lefthand side is 1 . In this way we rederive the value of $p$ as a condition for the global conservation of probability. Recall earlier that this condition was derived from purely local normalization of the probability distribution for a walker at a given point choosing its next step down one of the $z$ branches of the Cayley tree.

\section{Appendix B: The Transfer Matrix $\overrightarrow{\mathbf{\Psi}}$}

We determine the transfer matrix that connects the probability vectors $\overrightarrow{\mathrm{P}}_{\mathrm{i}+\mathrm{n}}$ and $\overrightarrow{\mathrm{P}}_{\mathrm{i}+\mathrm{m}+1}$ directly by comput- ing the connection between the vectors $\vec{P}_{i+1}$ and $\vec{P}_{i+2}$. From eq 53 we may immediately write down the vector $\overrightarrow{\mathrm{P}}_{\mathrm{i}+1}$ :

$$
\vec{P}_{i+1}=p\left(\begin{array}{l}
1 \\
z-2 \\
e^{-\Delta / T}
\end{array}\right)
$$

and we compute the vector $\vec{P}_{i+2}$ by considering the various cases. For example, if $\sigma_{i+1}$ is also in the forward direction (as defined by the ith bond vector), then there is a chance $p$ that $\sigma_{i+2}$ is also along the forward direction. The same probability applies if $\sigma_{i+1}$ is in one of the side directions. If, however, $\sigma_{i+1}$ is pointing backward, then the probability that $\sigma_{i+2}$ is in the forward direction is $\mathrm{pe}^{-\Delta /}$. Collecting these probabilities, we find

$$
\begin{aligned}
& P_{\text {front }}(i+2)=p P_{\text {front }}(i+1)+p P_{\text {side }}(i+1)+ \\
& \qquad e^{-\Delta / T} P_{\text {back }}(i+1)
\end{aligned}
$$

The equivalent expression for $P_{\text {back }}(i+2)$ is clearly analogous-we merely interchange the roles of forward and backward:

$$
\begin{array}{r}
P_{\text {back }}(i+2)=p P_{\text {back }}(i+1)+p P_{\text {side }}(i+1)+ \\
p e^{-\Delta / T} P_{\text {front }}(i+1)
\end{array}
$$

While, for the side, we must account for the fact that there are $z-2$ "side" directions by writing

$$
\begin{array}{r}
P_{\text {side }}(i+2)=p(z-2) P_{\text {front }}(i+1)+p[z-3+ \\
\left.e^{-\Delta / T}\right] P_{\text {side }}(i+1)+p P_{\text {front }}(i+1)
\end{array}
$$

The factor of $\left[z-3+\mathrm{e}^{-\Delta / T}\right]$ expresses the fact that if the $(i+1)$ th bond was al ong the side direction, there are $z-3$ other side directions of the $(i+2)$ th bond with no energetic penal ty associated and one side direction that constitutes a hairpin turn. Turning either to the forward or backward directions has the same probability and is not penalized.

Collecting these results in the form of

$$
\overrightarrow{\mathrm{P}}_{\mathrm{i}+2}=\overleftrightarrow{\Psi} \cdot \overrightarrow{\mathrm{P}}_{\mathrm{i}+1}
$$

allows us to read off the transfer matrix given in eq 54 .

\section{Appendix C: Solution to Discrete Recursion Relations: Generating Functions}

We now solve directly the discrete recursion relations, paying particular attention to the probability of deep retractions or, in other words, random walks that return near to the origin unentangled. By the introduction of generating functions, we are able to transform the recursion relations into algebraic equations that may then be solved for the generating functions of $P_{n, m}$ and $\mathrm{Q}_{n, m}$, respectively. To obtain these probability distributions from their respective generating functions, we will perform a contour integration. While the remaining contour integration cannot be done in closed form, we are able to determine the large $\mathrm{n}$ behavior of the probability distribution, keeping $\mathrm{m} \sim 0$ (1). 
To begin, we introduce the generating functions for $\mathrm{P}_{\mathrm{n}, \mathrm{m}}$ and $\mathrm{Q}_{\mathrm{n}, \mathrm{m}}$ below:

$$
\begin{aligned}
& p(x, t)=\sum_{m=0}^{\infty} \sum_{n=0}^{\infty} x^{m} t^{n} P_{n, m} \\
& q(x, t)=\sum_{m=0}^{\infty} \sum_{n=0}^{\infty} x^{m} t^{n} Q_{n, m}
\end{aligned}
$$

In eqs $\mathrm{C} 1$ and $\mathrm{C} 2$, the variables $\mathrm{x}$ and $\mathrm{t}$ are dual to the primitive path length $m$ and the total chain length $n$ respectively so they do not play the same roles that $x$ and $t$ did in the mapping of the problem onto the continuum advection-diffusion equation. Using the recursion relations, the boundary conditions, and initial conditions, we are able to solve for both $p(x, t)$ and $q(x, t)$. In the following we will concentrate only on the solution to $p(x, t)$-the solution for the generating function of the Q-walk distribution is found similarly.

After some lengthy algebra we may write the generating function for the P-walk distribution as

$$
p(x, t)=\frac{a(t)}{t} \frac{f_{1}(x, t)}{f_{2}(x, t)}-\frac{\alpha x^{2} t}{f_{2}(x, t)}
$$

where $\alpha$ was defined in eq 10 and the functions $f_{1}$ and $\mathrm{f}_{2}$ are defined below:

$$
\begin{gathered}
\mathrm{f}_{1}(\mathrm{x}, \mathrm{t})=\mathrm{t}^{2} \mathrm{x} \beta-\mathrm{x}+\mathrm{t}\left(1-\alpha+[\alpha-\beta] \mathrm{x}^{2}\right) \\
\mathrm{f}_{2}(\mathrm{x}, \mathrm{t})=\mathrm{t}(1-\alpha)-\mathrm{x}+\mathrm{x}^{2} \mathrm{t}(1-\beta)+\mathrm{t}^{2} \mathrm{x}(\alpha+\beta-1)
\end{gathered}
$$

with $\beta$ defined in eq 11 . The function of $\mathrm{t}$ called $\mathrm{a}(\mathrm{t})$ above is actually the generating function of $P_{n, 1}$ :

$$
a(t)=\sum_{n=0}^{\infty} P_{n, 1} t^{n}
$$

It may be checked in eq $C 3$ that $a(t)=\partial p /\left.\partial x\right|_{x=0}$ as selfconsistency demands. Both the solutions to $q(x, t)$ and $p(x, t)$ may be written in terms of this, as yet, unknown function $\mathrm{a}(\mathrm{t})$. To make progress, we must therefore calculate $a(t)$. We observe that the function $f_{2}$ has two roots $\left(x_{1}^{*}, x_{2}^{*}\right)$ in the complex $x$-plane, and consequently $\mathrm{p}(\mathrm{x}, \mathrm{t})$ has poles there. It may be shown that the product of the two roots is given by $x_{1}^{*} x_{2}^{*}=1 /(z-1)<1$ so at least one of these roots is inside the unit disk centered about the origin of the complex x-plane. Because the sum

$$
\sum_{m=0}^{\infty} P_{n, m}
$$

is convergent for all $\mathrm{n}, \mathrm{p}(\mathrm{x}, \mathrm{t})$ cannot have a pole within this unit disk since the double sum, given in eq $C 1$, is therefore convergent also. So we may determine a(t) by insisting that the smaller of the two poles, $\left(x_{1}^{*}, x_{2}^{*}\right)$ is, in fact, a removable singularity. We find that the generating function $a(t)$ is given by

$$
\frac{a(t)}{t}=\frac{\alpha\left[1+t^{2} \omega+k(t)\right]^{2}}{-2 \hat{\alpha}\left[1+t^{4} \alpha \omega-k(t)+t^{2}(\alpha k(t)-\hat{\beta}-2 \alpha \beta)\right]}
$$

where

$$
\mathrm{k}(\mathrm{t})=\sqrt{-4 \mathrm{t}^{2} \hat{\alpha} \hat{\beta}+\left(1+\mathrm{t}^{2} \omega\right)^{2}}
$$

and we have introduced the notation $\hat{y}=1-y$ for any $y$ and $\omega=1-\alpha-\beta$ in order to simplify the above formulas. The generating function for the probability of being a P-walk one step away from the origin is of the form $t g\left(t^{2}\right)$ (i.e., $a(t)$ is odd in $t$ ). This is expected since a walker can only be one step away from the origin after an odd number of time steps. The function a(t) can be expanded order by order in $t$ to recover the required probabilities $P_{n, 1}$ term by term, or one can write $P_{n, 1}$ in closed form as a contour integral. We will not, however, pursue that here but rather calculate $P_{n, m}$ in terms of a contour integral shortly. Combining eqs C3, $\mathrm{C} 8$, and C9, we arrive at the final form of the generating function for the probability distribution of P-walkers:

$$
p(x, t)=\frac{F(x, t)}{G(x, t)}
$$

where

$$
\begin{array}{r}
F(x, t)=-2 t^{2} \alpha+\frac{1}{\hat{\alpha}\left(1-t^{2}\right)}\left(-x+t\left[1+\left(x^{2}-1\right) \alpha\right]+\right. \\
(t-x) x \beta)\left(1-2 \alpha+-\omega t^{2}+\right. \\
\left.\sqrt{1+t^{4} \omega^{2}+2 t^{2}(\beta-\hat{\alpha}-2 \alpha \beta)}\right)
\end{array}
$$

and

$\mathrm{G}(\mathrm{x}, \mathrm{t})=-2\left(\mathrm{x}-\mathrm{t}\left(\hat{\alpha}+\mathrm{x}^{2} \hat{\beta}\right)\right)+\mathrm{t}^{2} \mathrm{x}(\hat{\alpha}-\beta)$

We now will replace $t$ by its conjugate variable, $n$, writing a new generating function as

$$
p_{n}(x)=\sum_{m=0}^{\infty} P_{n, m} x^{m}
$$

We perform this transformation by a contour integral in the complex t-plane:

$$
p_{n}(x)=\lim _{\epsilon \rightarrow 0} \frac{1}{2 \pi \mathrm{i}} \int \frac{p(x, t)}{|t|=\epsilon} \frac{t^{n+1}}{t^{n+1}}
$$

To perform this contour integral, we must examine the analytic structure of $p(x, t)$ in the complex $t$-plane. We find that there are two poles, $p_{1}$ and $p_{2}$, located at the roots of the polynomial:

$$
\mathrm{t}^{2} \mathrm{x} \omega-\mathrm{t}\left(\hat{\alpha}+\mathrm{x}^{2} \hat{\beta}\right)+\mathrm{x}=0
$$

and four branch points at the points $\pm \sqrt{b_{1,2}},\left|b_{2}\right|<\left|b_{1}\right|$ where

$$
\mathrm{b}_{1,2}=\frac{\omega+2 \alpha \beta \pm 2 \sqrt{\hat{\alpha} \hat{\beta}} \sqrt{\alpha \beta}}{\omega^{2}}
$$

In addition, there are two removable singularities located at $t= \pm 1$. All the nonanalyticities of the generating function lie on the real axis.

We first consider the two poles. The positions of the poles are a function of $x$. As we are interested in the Taylor series of $\mathrm{p}(\mathrm{x}, \mathrm{t})$ about the point $\mathrm{x}=0$, we consider the position of the poles in that limit only. The smaller of the two poles, $p_{1}$, goes to the origin as $x$ goes to zero but this is actually a removable singularity. Our 


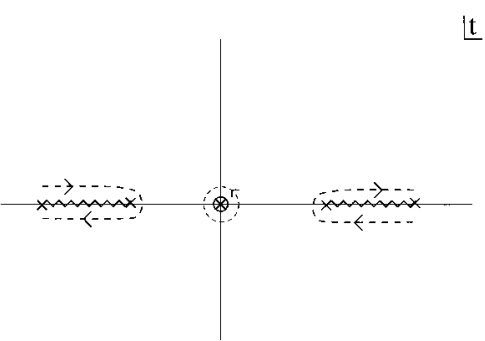

Figure 11. A sketch of the complex $t$ plane showing the original contour of integration about the origin and deformed contour of integration around the two symmetrically placed branch cuts.

calculation of a(t) assured this point. The second pole, $p_{2}$, goes to infinity as $1 / x$ in the limit of small $x$. Its contribution to the contour integral thus al so vanishes. We then turn to the branch points.

The branch points, unlike the poles, are independent of $\mathbf{x}$. They originate from a term of the form $|\omega|$ $\sqrt{\left(t^{2}-b_{1}\right)\left(t^{2}-b_{2}\right)}$ and the branch cuts may be drawn as shown in Figure 11. We are thus able to write $p_{n}(x)$ in terms of an integral over the difference in $p(x, t)$ across the two branch cuts. The integration contour, having been deformed from the loop encircling the origin (shown as a light dotted line in the figure) to one that goes around both branch cuts is shown as a heavy dashed line in the same figure. Thus, we find that we can write

$p_{n}(x)=\frac{1}{2 \pi \mathrm{i}} \int \frac{\sqrt{b_{1}} \frac{d t}{b_{2}} t^{n+1}}{n}\left[\Delta p(x, t)+(-1)^{n} \Delta p(x,-t)\right]$

where $\Delta \mathrm{p}(\mathrm{x}, \mathrm{t})$ is the difference in $\mathrm{p}(\mathrm{x}, \mathrm{t})$ across the branch cut.

While the limits of integration given by the branch points are not functions of $x$, they are functions of both $z$ and the hairpin energy $\Delta$. We note the behavior of these points in two limits of the hairpin energy. In the limit that $\Delta \rightarrow 0$ we find that $b_{1} \rightarrow \infty$ as $b_{1} \sim 4(z-1) / \Delta^{2}$ and $b_{2} \rightarrow z^{2} /(4(z-1))$ so the range of integration also diverges. In the opposite limit where $\Delta \rightarrow \infty$ we find that both $b_{2}$ and $b_{1}$ approach $z-1$ so the range of integration vanishes. The vanishing interval of integration al lows us to recover the large $\Delta$ form of the return probability which we argued heuristically in the text. The derivation of this result proceeds most simply from the calculation of $P_{n, 1}$ using a similar contour integration in the t-plane with the generating function a(t) discussed above. We find in the limit that $n e^{-\Delta / 2} \ll 1$ :

$$
\mathrm{P}_{\mathrm{n}, 1} \sim \frac{2}{3 \pi} \frac{\mathrm{e}^{-\Delta}}{(\mathrm{z}-1)^{\mathrm{n} / 2-1}} \sqrt{(\mathrm{z}-1)(\mathrm{z}-2)}
$$

This result has the expected dependence upon $\Delta$ and $n$, although the argument presented earlier applies most directly to the calculation of $Q_{n, 1}$ rather than $P_{n, 1}$ which was determined above.

From eq $\mathrm{C} 17$ it is clear that $\mathrm{p}_{n}(\mathrm{x})$ takes different forms, depending on whether $\mathrm{n}$ is odd or even. This distinction arises from the fact that a walker can only have a primitive path length which is odd after an odd number of time steps. We specialize in the remaining calculation to the case of odd $\mathrm{n}$ and find, after some algebra,

$$
\begin{aligned}
& p_{n}(x)=\frac{x \omega}{2 \pi} \int_{b_{2}}^{b_{1}} \frac{d u}{u^{(n+1) / 2}} x \\
& \frac{\left[x^{2}-u\left(\hat{\alpha}+\alpha x^{2}\right)\right] \sqrt{\left(b_{1}-u\right)\left(b_{2}-u\right)}}{\left[x^{2}\left(u^{2} \omega^{2}+1\right)-u\left(\hat{\alpha}^{2}+2 x^{2} \alpha \beta+x^{4} \hat{\beta}^{2}\right](u-1)\right.}
\end{aligned}
$$

Making the change of variables $u=b_{2} \exp (q)$ and using Watson's Lemma, ${ }^{19}$ we derive the first term of a large $n$ expansion of the above integral in which we keep $\mathrm{m} \sim 0$ (1). Finally, expanding in $\mathrm{x}$ we may write the solution as

$$
\begin{aligned}
P_{n, 2 m+1} \simeq & \frac{\omega}{\sqrt{2 \pi}} \frac{\sqrt{r-1}}{(n-1)^{3 / 2}} \frac{b_{2}^{-(n-3) / 2}}{b_{2}-1} \times \\
& \left\{\frac{1}{b_{2} \hat{\beta}^{2}} \frac{1}{\kappa_{2}-\kappa_{1}}\left(\frac{\gamma_{1}+\gamma_{2} \kappa_{1}}{\kappa_{1}^{m+1}}-\frac{\gamma_{1}+\gamma_{2} \kappa_{2}}{\kappa_{2}^{m+1}}\right)\right\}
\end{aligned}
$$

where we have introduced the following quantities: $\gamma_{1}$ $=\hat{\alpha} b_{2}, \gamma_{2}=b_{2} \alpha-1, \kappa_{1}=A-\sqrt{A^{2}-B}$, and $\kappa_{2}=A+$ $\sqrt{A^{2}-B}$ where

$$
\begin{gathered}
A=\frac{1-b_{2} \alpha \beta+b_{2}^{2} \omega^{2}}{2 b_{2} \hat{\beta}^{2}} \\
B=\left(\frac{\hat{\alpha}}{\hat{\beta}}\right)^{2}
\end{gathered}
$$

The above analytic result supports the numerical determination of the arm potential for deep retraction discussed in the text.

\section{References and Notes}

(1) Ferry, J. D. Viscoelastic Properties of Polymers, 3rd ed.; Wiley: New York, 1980.

(2) Graessley, W. W. Macromolecules 1982, 15, 1164; Carallea, J . M.; Gotro, J . T.; Graessley, W. W. Macromolecules 1986, $19,659$.

(3) Fetters, L.J .; Kiss, A. D.; Pearson, D. S.; Quack, G. F.; Vitus, F. J . Macromolecules 1993, 26, 647.

(4) Raju, V. R.; Rachapudy, H.; Graessley, W. W. J . Polym. Sci.: Polym. Phys. 1979, 17, 1223.

(5) Raju, V. R.; Graessley, W. W. J . Polym. Sci.: Polym. Symp. 1984, 71, 77.

(6) Raju, V. R. Ph.D. Dissertation, Princeton University, 1980.

(7) Edwards, S. F. Proc. Phys. Soc. 1967, 92, 9.

(8) de Gennes, P.-G. J . Chem. Phys. 1971, 55, 572.

(9) Pearson, D. S.; Helfand, E. Macromolecules 1984, 17, 888.

(10) Ball, R. C.; McLeish, T. C. B. Macromolecules 1989, 22, 1911.

(11) Milner, S. T.; McLeish, T. C. B. Macromolecules 1997, 30, 2159.

(12) Fetters, L. J .; Graessley, W. W.; Krishnamoorti, R.; Lohse, D. J . Macromolecules 1997, 30, 4973.

(13) Nechaev, S. K.; Semenov, A. N.; Koleva, M. K. Physica 1987, 140A, 506.

(14) Khokhlov, A. R.; Nechaev, S. K. Phys. Lett. 1985, 112A, 156

(15) Helfand, E.; Pearson, D. S. J . Chem. Phys. 1983, 79, 2054.

(16) Yurasova, T. A.; McLeish, T. C. B.; Semenov, A. N. Macromolecules 1994, 27, 7205.

(17) Doi, M.; Edwards, S. F. The Theory of Polymer Dynamics; Oxford University Press: New York, 1986.

(18) Milner, S. T. J . Rheol. 1996, 40, 303.

(19) Copson, E. T. CambridgeTracts in Mathematics: Asymptotic Expansions; Cambridge University Press: Cambridge, 1965. MA980347A 